\title{
Image singularities of Green's functions for isotropic elastic bimaterials subjected to concentrated forces and dislocations
}

\author{
Chien-Ching $\mathrm{Ma}^{*}$, Ru-Li Lin \\ Department of Mechanical Engineering, National Taiwan University, Taipei 10617, Taiwan, ROC
}

Received 17 November 2001; received in revised form 4 July 2002

\begin{abstract}
Green's functions for isotropic materials in the two-dimensional problem for elastic bimaterials with perfectly bonded interface are reexamined in the present study. Although the Green's function for an isotropic elastic bimaterial subjected to a line force or a line dislocation has been discussed by many authors, the physical meaning and the structure of the solution are not clear. In this investigation, the Green's function for an elastic bimaterial is shown to consist of eight Green's functions for a homogeneous infinite plane. One of the novel features is that Green's functions for bimaterials can be expressed directly by knowing Green's functions for the infinite plane. If the applied load is located in material 1, the solution for the half-plane of material 1 is constructed with the help of five Green's functions corresponding to the infinite plane. However, the solution for the half-plane of material 2 only consists of three Green's functions for the infinite plane. One of the five Green's functions of material 1 and all the three Green's functions of material 2 have their singularities located in the half-plane where the load is applied, and the other four image singularities of material 1 are located outside the half-plane at the same distance from the interface as that of the applied load. The nature and magnitude of the image singularities for both materials are presented explicitly from the principle of superposition, and classified according to different loads. It is known that for the problem of anisotropic bimaterials subjected to concentrated forces and dislocations, the image singularities are simply concentrated forces and dislocations with the stress singularity of order $\mathrm{O}(1 / r)$. However, higher orders $\left(\mathrm{O}\left(1 / r^{2}\right)\right.$ and $\left.\mathrm{O}\left(1 / r^{3}\right)\right)$ of stress singularities are found to exist in this study for isotropic bimaterials. The highest order of the stress singularity is $\mathrm{O}\left(1 / r^{3}\right)$ for the image singularities of material 1 , and is $\mathrm{O}\left(1 / r^{2}\right)$ for material 2. Using the present solution, Green's functions associated with the problems of elastic half-plane with free and rigidly fixed boundaries, for homogeneous isotropic elastic solid, are obtained as special cases. (C) 2002 Elsevier Science Ltd. All rights reserved.
\end{abstract}

Keywords: Image singularities; Isotropic bimaterials; Concentrated forces; Dislocations; Image point; Object point

\section{Introduction}

The importance of Green's functions in constructing solutions to boundary value problems has been well recognized. Thus, the fundamental solutions involving Green's functions of half-plane and bimaterial have

\footnotetext{
${ }^{*}$ Corresponding author. Tel.: +886-2-2365-9996; fax: +886-2-2363-1755.

E-mail address: cma@w3.me.ntu.edu.tw (C.-C. Ma).
} 
been obtained by many different methods. Green's function for two-dimensional deformations of an anisotropic elastic half-space subjected to a line force and/or a line dislocation inside the half-space has been considered by Willis (1970), Barnett and Lothe (1974), Suo (1990) and Qu and Li (1991). In the earlier study of Green's function for the half-space with a traction-free boundary condition, the solution is constructed from Green's function for the infinite space by adding a distribution of forces on the half-space surface so that the net surface traction vanishes. With this approach, the solution is not explicit and the final result requires an integration of the distributed forces on the surface. Some progress in constructing Green's function has been made by Hwu and Yen (1991) and Ting (1992).

The method of image is a technique that uses the superposition of known solution to construct the solution to other complicated problems. The point where the applied force is located is called "object" point, and the point situated on the opposite side of the global axes with respect to the object point is referred to as "image" point. In the theory of linear elasticity, the two-dimensional isotropic anti-plane problems can be solved by a superposition of some simple image singularities over the plane. Similar methods can also be applied to the two-dimensional anisotropic plane and anti-plane problems, see for instance Ting (1996). The image singularities of Green's functions for anisotropic elastic half-plane and bimaterials were discussed in detail by Ting (1992). When an anisotropic half-plane is subjected to a concentrated force or dislocation within the bimaterial, the image singularities are simply forces and dislocations. In general, the locations of image singularities of Green's functions for an anisotropic half-plane are different, and depend on the anisotropic elastic constants. There are at most nine image points located at different positions, which are generally not at equidistant points in relation to the object point. As is well known for the isotropic half-plane, which is the degenerate case of anisotropic materials, the image singularities are not merely concentrated forces and dislocations (Ma and Lin, 2001). Higher order image singularities (i.e. double force, double moment etc.) may exist for the isotopic material, and the nine image points coalesce into a single point which is at an equidistant location with respect to the object point. The object in this paper is to find the image singularities existing at the image points for isotropic bimaterials, and to provide physical meanings of the Green's function.

There is an interesting group of solutions for the infinite space of linear isotropic elastic materials, which are referred to as the nuclei of strain solutions. The solutions of many interesting problems may be derived in terms of combinations of these nuclei or by a process of superposition of nuclei. The fundamental solution for the three-dimensional deformation of an infinite isotropic elastic body subjected to a point force is well known as the Kelvin solution. By the differentiation of this solution, a family of additional nuclei can be constructed, such as the double force, and the double force with moment. Mindlin's (1936) results for a point force in the interior of an elastic half-space are based on the consideration of nuclei of strain. Such solutions may be termed as half-space nuclei of strain. However, some of the nuclei of strain have the form of a line extending from a fixed distance to infinity with constant or variable magnitude. There have been several investigations and applications on this subject following Mindlin's study. Mindlin and Cheng (1950) provided many basic solutions for the nuclei of strain in the half-space solid. The case of an elastic half-space with a fixed boundary has been considered by PhanThien (1983) using Mindlin's approach. Green's function for the axisymmetric problem of a bimaterial elastic solid has been investigated by Hasegawa et al. (1992). The fundamental solutions for point forces in the interior of one of two elastic half-spaces joined by a sliding contact interface was given by Dundurs and Hetenyi (1965). Vijayakumar and Cormack (1987a,b) presented a general approach to derive Green's function for three-dimensional bimaterial elastic media with bonded and sliding interfaces by dividing the nuclei into independent classes, and by representing the displacements and stresses produced by these nuclei within the framework of matrix-vector operations. Further developments were made by Carvalho and Curran (1992) who obtained the two-dimensional Green's functions for plane strain in elastic isotropic bimaterials by reducing the three-dimensional nuclei of strain through an integral procedure. The complete solution for the stresses produced by the application of a concentrated force in the interior of an 
elastic bimaterial was obtained by Ma and Huang (1996), using the transient wave analysis and taking appropriate limits.

This paper investigates Green's functions for a concentrated force or a dislocation in the interior of an isotropic elastic bimaterial for both plane strain and plane stress cases. The aim of this paper is to study the Green's function of a bimaterial and to discuss in detail the structure of singularities on the image point. There are eight kinds of image singularity in a half-plane problem involving applied force and dislocation, namely single force, single dislocation, double force without moment, double force with moment, center of extension, center of shear, double center of expansion, and double moment, as discussed by Ma and Lin (2001). It is interesting to note that a dislocation is one of the image singularities for a traction free half-plane subjected to concentrated forces, and a concentrated force is one of the image singularities for a rigidly fixed half-plane subjected to dislocations. This paper extends the results for the half-plane problem obtained by Ma and Lin (2001) to the more general bimaterial case. It is found that besides the above mentioned image singularities for the half-plane problem, there are three new image singularities arising in the bimaterial problem, namely concentrated moment, center of dilatation and center of expansion. Since all the solutions of higher order image singularities (i.e. double force, double moment etc.) can be constructed by the differentiation of the solutions for force and dislocation, Green's functions for a bimaterial are determined entirely from the solutions of force and dislocation in the infinite plane.

\section{Green's functions and image singularities for bimaterials}

\subsection{General formulation}

Consider a two-dimensional isotropic bimaterial with a straight perfectly bonded interface at $y=0$. Fig. 1a shows two dissimilar elastic solids with shear modulus $\mu_{i}$ and Poisson's ratio $v_{i}(i=1,2)$ subjected to a concentrated force or dislocation applied at $y=h$ in material 1. Let material $1(i=1)$ and material $2(i=2)$ occupy the half-plane $y \geqslant 0$ and $y \leqslant 0$, respectively.

For the two-dimensional in-plane problem, the stresses $\sigma_{i j}$ and displacements $u_{i}$ only depend on $x$ and $y$. The equations of equilibrium can be written as

$$
\sigma_{j x, x}+\sigma_{j y, y}=0, \quad j=x, y,
$$

where the comma denotes partial differentiation. The stress functions $\phi_{j}$ (Ting, 1996) are introduced such that

$$
\sigma_{j x}=-\frac{\partial \phi_{j}}{\partial y}, \quad \sigma_{j y}=\frac{\partial \phi_{j}}{\partial x}
$$

Then the equations of equilibrium are automatically satisfied and $\phi_{j}$ are determined by satisfying the compatibility equation and boundary conditions. It is sufficient therefore to determine the stress functions $\phi_{j}$, since the stresses can then be obtained from (2.2). With the usual approach of the method of image, the problem of bimaterials is reduced to two independent infinite plane problems, involving materials 1 and 2, the only necessary constrain being that Green's functions for the two infinite planes must satisfy the interface continuity conditions. Then the complete Green's functions for the stress functions and displacements for materials 1 and 2 can be obtained by considering the following two problems: (i) an infinite plane of material 1 subjected to applied singularities at the object point $y=h$, and image singularities at the image point $y=-h$, see Fig. 1b; (ii) an infinite plane of material 2 subjected to image singularities at the image point $y=h$, see Fig. 1c. The solutions to the stress functions and displacements can be expressed by the principle of superposition as 


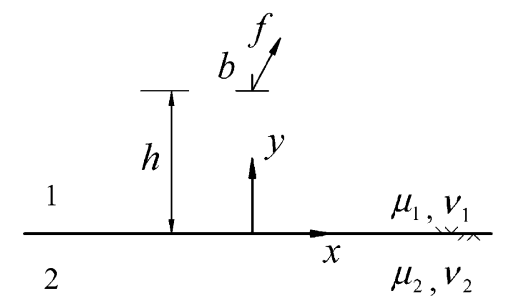

(a)

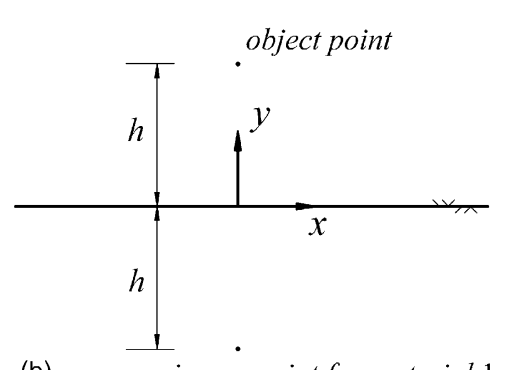

(b)

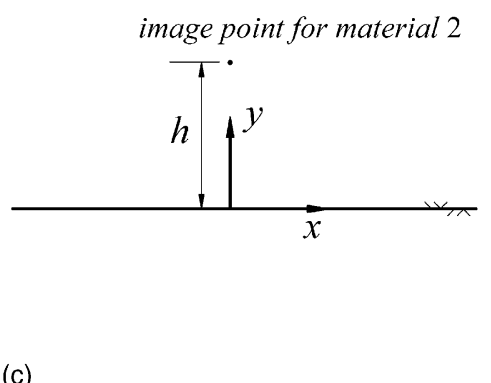

(c)

Fig. 1. (a) An elastic isotropic bimaterial subjected to a concentrated force and dislocation. (b) The image and object points for material 1. (c) The image point for material 2.

$$
\phi_{j}^{1}=\phi_{j}^{\mathrm{o}}+\phi_{j}^{\mathrm{i}}, \quad u_{j}^{1}=u_{j}^{\mathrm{o}}+u_{j}^{\mathrm{i}}, \quad j=x, y,
$$

for material 1 and

$$
\phi_{j}^{2}=\phi_{j}^{\mathrm{i}}, \quad u_{j}^{2}=u_{j}^{\mathrm{i}}, \quad j=x, y,
$$

for material 2, where $u_{j}$ are the in-plane displacements. The superscripts "o" and "i" denote the force or dislocation which acts at the object and image point of the infinite plane, respectively. The force or dislocation systems acting at the object and image points are referred to as the applied singularities and the image singularities, respectively. The image points of materials 1 and 2 are evidently different, and are always located at the same distance from the interface for isotropic materials. It should be noted that the image point for material 2 is the same as the object point for material 1 . The first term on the right-hand side of (2.3) represents Green's function for an infinite plane with a concentrated force or a dislocation applied at the object point. The second term is the disturbed solution which is added at the image point to satisfy the continuity conditions at the interface. Hence, Green's function for material 1 includes the applied singularities and image singularities on the object and image points of the infinite plane. However, Green's function for material 2 only includes image singularities at the image point of the infinite plane. The structure and magnitude of the image singularities for the half-planes of material 1 and 2 with different applied singularities will be discussed in detail in this paper. We begin with the simple case of a vertical concentrated force, for which the solution will be discussed in the following section.

\subsection{Image singularities of bimaterials subjected to a vertical concentrated force $f_{y}$}

A set of Cartesian coordinates is introduced at the interface of a bimaterial, where the $x$-axis is taken along the interface. A vertical concentrated force of magnitude $f_{y}$ is applied at the point $x=0, y=h$, in the positive $y$-direction in the material 1 . The continuity conditions for traction and displacement along the interface are

$$
\begin{aligned}
& \phi_{x}^{1}(x, 0)=\phi_{x}^{2}(x, 0), \quad \phi_{y}^{1}(x, 0)=\phi_{y}^{2}(x, 0), \\
& u_{x}^{1}(x, 0)=u_{x}^{2}(x, 0), \quad u_{y}^{1}(x, 0)=u_{y}^{2}(x, 0) .
\end{aligned}
$$

The superscripts " 1 " and " 2 " indicate materials 1 and 2 , respectively. It is convenient to use stress functions to solve the problem rather than the stresses themselves. We will explain in some detail the construction of 
the result for the image singularities of materials 1 and 2 . The fundamental solutions for the stress functions and displacements of an infinite plane of material 1, subjected to a vertical force of magnitude $f_{y}$ at the object point $x=0, y=h$, (reduced from the solution given by Ting (1996) for the anisotropic case, p. 247), are

$$
\begin{aligned}
& \phi_{x}^{1}=\frac{f_{y}}{\pi\left(1+k_{1}\right)}\left[\frac{(y-h)^{2}}{x^{2}+(y-h)^{2}}+\frac{1-k_{1}}{2} \ln \sqrt{x^{2}+(y-h)^{2}}\right], \\
& \phi_{y}^{1}=\frac{f_{y}}{\pi\left(1+k_{1}\right)}\left[\frac{1+k_{1}}{2} \tan ^{-1} \frac{y-h}{x}-\frac{x(y-h)}{x^{2}+(y-h)^{2}}\right], \\
& u_{x}^{1}=\frac{f_{y}}{2 \pi \mu_{1}\left(1+k_{1}\right)} \frac{x(y-h)}{x^{2}+(y-h)^{2}}, \\
& u_{y}^{1}=\frac{f_{y}}{2 \pi \mu_{1}\left(1+k_{1}\right)}\left[\frac{(y-h)^{2}}{x^{2}+(y-h)^{2}}-k_{1} \ln \sqrt{x^{2}+(y-h)^{2}}\right],
\end{aligned}
$$

where

$$
k_{i}=\left\{\begin{array}{ll}
3-4 v_{i}, & \text { for plane strain } \\
\frac{3-v_{i}}{1+v_{i}}, & \text { for plane stress }
\end{array}, \quad i=1,2 .\right.
$$

Because of the two special functions $\ln ()$ and $\tan ^{-1}()$ appearing in Eq. (2.6), it is necessary to add another solution to establish the interface continuity, so that terms involving $\ln ()$ and $\tan ^{-1}()$ for material 1 become identical to those for material 2 along $y=0$. The only way to achieve this is to apply image singularities that have the same special functions as indicated in Eq. (2.6). Hence the solution to image singularities for material 1 is considered as follows:

$$
\begin{aligned}
\phi_{x}^{1} & =A_{1} \frac{(y+h)^{2}}{x^{2}+(y+h)^{2}}+B_{1} \ln \left(x^{2}+(y+h)^{2}\right), \\
\phi_{y}^{1} & =-A_{1} \frac{x(y+h)}{x^{2}+(y+h)^{2}}+\left(A_{1}-2 B_{1}\right) \tan ^{-1} \frac{y+h}{x}, \\
u_{x}^{1} & =\frac{-1}{2 \mu_{1}}\left[\left(\frac{1-k_{1}}{2} A_{1}-2 B_{1}\right) \tan ^{-1} \frac{y+h}{x}-A_{1} \frac{x(y+h)}{x^{2}+(y+h)^{2}}\right], \\
u_{y}^{1} & =\frac{1}{2 \mu_{1}}\left[A_{1} \frac{(y+h)^{2}}{x^{2}+(y+h)^{2}}+\left(B_{1}-\frac{1+k_{1}}{4} A_{1}\right) \ln \left(x^{2}+(y+h)^{2}\right)\right] .
\end{aligned}
$$

It is worth noting that the expression in (2.7) have the general form for the solution corresponding to a vertical force of magnitude $f_{y}$ at $x=0, y=-h$, as well as to a dislocation with horizontal Burger's vector $b_{x}$ at $x=0, y=-h$. The unknown coefficients $A_{1}$ and $A_{2}$ will be determined later.

In order to satisfy the continuity condition at the interface, we must apply a similar type of solution to the image singularity at the image point for material 2 . This solution may be expressed as 


$$
\begin{aligned}
& \phi_{x}^{2}=A_{2} \frac{(y-h)^{2}}{x^{2}+(y-h)^{2}}+B_{2} \ln \left(x^{2}+(y-h)^{2}\right), \\
& \phi_{y}^{2}=-A_{2} \frac{x(y-h)}{x^{2}+(y-h)^{2}}+\left(A_{2}-2 B_{2}\right) \tan ^{-1} \frac{y-h}{x}, \\
& u_{x}^{2}=\frac{-1}{2 \mu_{2}}\left[\left(\frac{1-k_{2}}{2} A_{2}-2 B_{2}\right) \tan ^{-1} \frac{y-h}{x}-A_{2} \frac{x(y-h)}{x^{2}+(y-h)^{2}}\right], \\
& u_{y}^{2}=\frac{1}{2 \mu_{2}}\left[A_{2} \frac{(y-h)^{2}}{x^{2}+(y-h)^{2}}+\left(B_{2}-\frac{1+k_{2}}{4} A_{2}\right) \ln \left(x^{2}+(y-h)^{2}\right)\right],
\end{aligned}
$$

where $A_{2}$ and $B_{2}$ are unknown coefficients.

Setting $y=0$, and substituting (2.6)-(2.8) into (2.5), and collecting the terms involving $\ln ()$ and $\tan ^{-1}()$ along the interface, four equations for four unknown constants $A_{1}, A_{2}, B_{1}$ and $B_{2}$ are obtained as follows:

$$
\begin{aligned}
& B_{1}+\frac{\left(1-k_{1}\right) f_{y}}{4 \pi\left(1+k_{1}\right)}=B_{2}, \\
& \left(A_{1}-2 B_{1}\right)-\frac{f_{y}}{2 \pi}=2 B_{2}-A_{2}, \\
& \frac{1}{2 \mu_{1}}\left(2 B_{1}-\frac{1-k_{1}}{2} A_{1}\right)=\frac{1}{2 \mu_{2}}\left(\frac{1-k_{2}}{2} A_{2}-2 B_{2}\right), \\
& \frac{1}{2 \mu_{1}}\left(B_{1}-\frac{1+k_{1}}{4} A_{1}\right)-\frac{k_{1} f_{y}}{4 \pi \mu_{1}\left(1+k_{1}\right)}=\frac{1}{2 \mu_{2}}\left(B_{2}-\frac{1+k_{2}}{4} A_{2}\right) .
\end{aligned}
$$

The linear system of Eq. (2.9) are solved to obtain $A_{1}, B_{1}, A_{2}$ and $B_{2}$ explicitly as follows:

$$
\begin{aligned}
& A_{1}=\frac{(1-v) k_{1}}{\pi\left(1+k_{1}\right)\left(1+v k_{1}\right)} f_{y}, \quad B_{1}=\frac{\left(k_{1}-1\right)\left(k_{1} v^{2}+k_{2}\right)+2 k_{1} v\left(1-k_{2}\right)}{4 \pi\left(1+k_{1}\right)\left(1+v k_{1}\right)\left(v+k_{2}\right)} f_{y}, \\
& A_{2}=\frac{v}{\pi\left(v+k_{2}\right)} f_{y}, \quad B_{2}=\frac{\left(1-k_{1} k_{2}\right) v}{4 \pi\left(v+k_{2}\right)\left(1+v k_{1}\right)} f_{y},
\end{aligned}
$$

where $v=\mu_{2} / \mu_{1}$ is the ratio of the two shear moduli. The remaining terms, which give rise to discontinuities along the interface, are

$$
\begin{aligned}
\phi_{x}^{1} & =A_{1} \frac{h^{2}}{x^{2}+h^{2}}+\frac{f_{y}}{\pi\left(1+k_{1}\right)} \frac{h^{2}}{x^{2}+h^{2}}, \\
\phi_{y}^{1} & =-A_{1} \frac{x h}{x^{2}+h^{2}}+\frac{f_{y}}{\pi\left(1+k_{1}\right)} \frac{x h}{x^{2}+h^{2}}, \\
u_{x}^{1} & =\frac{A_{1}}{2 \mu_{1}} \frac{x h}{x^{2}+h^{2}}-\frac{f_{y}}{2 \pi \mu_{1}\left(1+k_{1}\right)} \frac{x h}{x^{2}+h^{2}}, \\
u_{y}^{1} & =\frac{A_{1}}{2 \mu_{1}} \frac{h^{2}}{x^{2}+h^{2}}+\frac{f_{y}}{2 \pi \mu_{1}\left(1+k_{1}\right)} \frac{h^{2}}{x^{2}+h^{2}},
\end{aligned}
$$

for material 1 , and

$$
\begin{aligned}
& \phi_{x}^{2}=A_{2} \frac{h^{2}}{x^{2}+h^{2}}, \quad \phi_{y}^{2}=A_{2} \frac{x h}{x^{2}+h^{2}}, \\
& u_{x}^{2}=\frac{-A_{2}}{2 \mu_{2}} \frac{x h}{x^{2}+h^{2}}, \quad u_{y}^{2}=\frac{A_{2}}{2 \mu_{2}} \frac{h^{2}}{x^{2}+h^{2}},
\end{aligned}
$$


for material 2. Now, $A_{1}$ and $A_{2}$ are known values as indicated in (2.10). The stresses functions expressed in (2.11) and (2.12) have the $1 / r^{2}$ and $1 / r^{3}$ types of stress singularities, where $r$ is the radial distance from the image point. Thus, another suitable fundamental solutions with the stress singularity $1 / r^{2}$ are chosen to superpose at the image points of material 1 and 2. The proposed solutions are

$$
\begin{aligned}
\phi_{x}^{1} & =I_{1} \frac{y+h}{x^{2}+(y+h)^{2}}+J_{1} \frac{x^{2}(y+h)}{\left(x^{2}+(y+h)^{2}\right)^{2}}, \\
\phi_{y}^{1} & =J_{1} \frac{x(y+h)^{2}}{\left(x^{2}+(y+h)^{2}\right)^{2}}-I_{1} \frac{x}{x^{2}+(y+h)^{2}}, \\
u_{x}^{1} & =\frac{1}{2 \mu_{1}}\left[\left(I_{1}+\frac{1+k_{1}}{4} J_{1}\right) \frac{x}{x^{2}+(y+h)^{2}}-J_{1} \frac{x(y+h)^{2}}{\left(x^{2}+(y+h)^{2}\right)^{2}}\right], \\
u_{y}^{1} & =\frac{1}{2 \mu_{1}}\left[\left(I_{1}-\frac{1+k_{1}}{4} J_{1}\right) \frac{(y+h)^{3}}{\left(x^{2}+(y+h)^{2}\right)^{2}}+\left(I_{1}+\frac{3-k_{1}}{4} J_{1}\right) \frac{x^{2}(y+h)}{\left(x^{2}+(y+h)^{2}\right)^{2}}\right],
\end{aligned}
$$

for material 1 , and

$$
\begin{aligned}
\phi_{x}^{2} & =I_{2} \frac{y-h}{x^{2}+(y-h)^{2}}+J_{2} \frac{x^{2}(y-h)}{\left(x^{2}+(y-h)^{2}\right)^{2}}, \\
\phi_{y}^{2} & =J_{2} \frac{x(y-h)^{2}}{\left(x^{2}+(y-h)^{2}\right)^{2}}-I_{2} \frac{x}{x^{2}+(y-h)^{2}}, \\
u_{x}^{2} & =\frac{1}{2 \mu_{2}}\left[\left(I_{2}+\frac{1+k_{2}}{4} J_{2}\right) \frac{x}{x^{2}+(y-h)^{2}}-J_{2} \frac{x(y-h)^{2}}{\left(x^{2}+(y-h)^{2}\right)^{2}}\right], \\
u_{y}^{2} & =\frac{1}{2 \mu_{2}}\left[\left(I_{2}-\frac{1+k_{2}}{4} J_{2}\right) \frac{(y-h)^{3}}{\left(x^{2}+(y-h)^{2}\right)^{2}}+\left(I_{2}+\frac{3-k_{2}}{4} J_{2}\right) \frac{x^{2}(y-h)}{\left(x^{2}+(y-h)^{2}\right)^{2}}\right],
\end{aligned}
$$

for material 2, where $I_{1}, I_{2}, J_{1}$ and $J_{2}$ are unknown coefficients. The expressions (2.13) and (2.14) are the general solutions for applying the double force without moment along the $x$-direction and the center of dilatation at the image points of material 1 and 2, respectively. The stress functions shown in (2.13) and (2.14) have the $1 / r^{2}$ order of stress singularity. Setting $y=0$ in (2.13) and (2.14), adding them to (2.11) and (2.12), respectively, the stress functions and displacements at the interface are found to be

$$
\begin{aligned}
& \phi_{x}^{1}=A_{1} \frac{h^{2}}{x^{2}+h^{2}}+\frac{f_{y}}{\pi\left(1+k_{1}\right)} \frac{h^{2}}{x^{2}+h^{2}}+I_{1} \frac{h}{x^{2}+h^{2}}+J_{1} \frac{x^{2} h}{\left(x^{2}+h^{2}\right)^{2}}, \\
& \phi_{y}^{1}=\left(-A_{1} h+\frac{f_{y} h}{\pi\left(1+k_{1}\right)}\right) \frac{x}{x^{2}+h^{2}}+J_{1} \frac{x h^{2}}{\left(x^{2}+h^{2}\right)^{2}}-I_{1} \frac{x}{x^{2}+h^{2}}, \\
& u_{x}^{1}=\frac{1}{2 \mu_{1}}\left[A_{1} h-\frac{f_{y} h}{\pi\left(1+k_{1}\right)}+\left(I_{1}+\frac{1+k_{1}}{4} J_{1}\right)\right] \frac{x}{x^{2}+h^{2}}-\frac{J_{1}}{2 \mu_{1}} \frac{x h^{2}}{\left(x^{2}+h^{2}\right)^{2}}, \\
& u_{y}^{1}=\frac{1}{2 \mu_{1}}\left(A_{1}+\frac{f_{y}}{\pi\left(1+k_{1}\right)}\right) \frac{h^{2}}{x^{2}+h^{2}}+\frac{1}{2 \mu_{1}}\left[\left(I_{1}-\frac{1+k_{1}}{4} J_{1}\right) \frac{h^{3}}{\left(x^{2}+h^{2}\right)^{2}}+\left(I_{1}+\frac{3-k_{1}}{4} J_{1}\right) \frac{x^{2} h}{\left(x^{2}+h^{2}\right)^{2}}\right],
\end{aligned}
$$


for material 1 , and

$$
\begin{aligned}
& \phi_{x}^{2}=A_{2} \frac{h^{2}}{x^{2}+h^{2}}-I_{2} \frac{h}{x^{2}+h^{2}}-J_{2} \frac{x^{2} h}{\left(x^{2}+h^{2}\right)^{2}} \\
& \phi_{y}^{2}=A_{2} \frac{x h}{x^{2}+h^{2}}+J_{2} \frac{x h^{2}}{\left(x^{2}+h^{2}\right)^{2}}-I_{2} \frac{x}{x^{2}+h^{2}} \\
& u_{x}^{2}=\frac{-A_{2}}{2 \mu_{2}} \frac{x h}{x^{2}+h^{2}}+\frac{1}{2 \mu_{2}}\left[\left(I_{2}+\frac{1+k_{2}}{4} J_{2}\right) \frac{x}{x^{2}+h^{2}}-J_{2} \frac{x h^{2}}{\left(x^{2}+h^{2}\right)^{2}}\right], \\
& u_{y}^{2}=\frac{A_{2}}{2 \mu_{2}} \frac{h^{2}}{x^{2}+h^{2}}-\frac{1}{2 \mu_{2}}\left[\left(I_{2}-\frac{1+k_{2}}{4} J_{2}\right) \frac{h^{3}}{\left(x^{2}+h^{2}\right)^{2}}+\left(I_{2}+\frac{3-k_{2}}{4} J_{2}\right) \frac{x^{2} h}{\left(x^{2}+h^{2}\right)^{2}}\right]
\end{aligned}
$$

for material 2. Imposing the condition of continuity of the terms with $1 / r^{2}$ type of stress singularities at the interface, we have

$$
\begin{aligned}
& -A_{1} h+\frac{f_{y} h}{\pi\left(1+k_{1}\right)}-I_{1}=A_{2} h-I_{2}, \\
& v A_{1} h-\frac{v f_{y} h}{\pi\left(1+k_{1}\right)}+v\left(I_{1}+\frac{1+k_{1}}{4} J_{1}\right)=-A_{2} h+I_{2}+\frac{1+k_{2}}{4} J_{2} .
\end{aligned}
$$

Now, the remaining stress functions and displacements at the interface are

$$
\begin{aligned}
\phi_{x}^{1} & =A_{1} \frac{h^{2}}{x^{2}+h^{2}}+\frac{f_{y}}{\pi\left(1+k_{1}\right)} \frac{h^{2}}{x^{2}+h^{2}}+I_{1} \frac{h}{x^{2}+h^{2}}+J_{1} \frac{x^{2} h}{\left(x^{2}+h^{2}\right)^{2}}, \\
\phi_{y}^{1} & =J_{1} \frac{x h^{2}}{\left(x^{2}+h^{2}\right)^{2}}, \\
u_{x}^{1} & =\frac{-J_{1}}{2 \mu_{1}} \frac{x h^{2}}{\left(x^{2}+h^{2}\right)^{2}}, \\
u_{y}^{1} & =\frac{1}{2 \mu_{1}}\left(A_{1}+\frac{f_{y}}{\pi\left(1+k_{1}\right)}\right) \frac{h^{2}}{x^{2}+h^{2}}+\frac{1}{2 \mu_{1}}\left[\left(I_{1}-\frac{1+k_{1}}{4} J_{1}\right) \frac{h^{3}}{\left(x^{2}+h^{2}\right)^{2}}+\left(I_{1}+\frac{3-k_{1}}{4} J_{1}\right) \frac{x^{2} h}{\left(x^{2}+h^{2}\right)^{2}}\right],
\end{aligned}
$$

for material 1 , and

$$
\begin{aligned}
& \phi_{x}^{2}=A_{2} \frac{h^{2}}{x^{2}+h^{2}}-I_{2} \frac{h}{x^{2}+h^{2}}-J_{2} \frac{x^{2} h}{\left(x^{2}+h^{2}\right)^{2}} \\
& \phi_{y}^{2}=J_{2} \frac{x h^{2}}{\left(x^{2}+h^{2}\right)^{2}} \\
& u_{x}^{2}=\frac{-J_{2}}{2 \mu_{2}} \frac{x h^{2}}{\left(x^{2}+h^{2}\right)^{2}} \\
& u_{y}^{2}=\frac{A_{2}}{2 \mu_{2}} \frac{h^{2}}{x^{2}+h^{2}}-\frac{1}{2 \mu_{2}}\left[\left(I_{2}-\frac{1+k_{2}}{4} J_{2}\right) \frac{h^{3}}{\left(x^{2}+h^{2}\right)^{2}}+\left(I_{2}+\frac{3-k_{2}}{4} J_{2}\right) \frac{x^{2} h}{\left(x^{2}+h^{2}\right)^{2}}\right]
\end{aligned}
$$

for material 2. It is remarkable to note that every term in (2.18) and (2.19) has $1 / r^{3}$ type of stress singularity. We therefore apply a suitable image singularity with $1 / r^{3}$ type of stress singularity only at the image point of material 1 , the corresponding solution being 


$$
\begin{aligned}
& \phi_{x}^{1}=E \frac{x^{2}-(y+h)^{2}}{\left(x^{2}+(y+h)^{2}\right)^{2}}, \quad \phi_{y}^{1}=E \frac{2 x(y+h)}{\left(x^{2}+(y+h)^{2}\right)^{2}}, \\
& u_{x}^{1}=\frac{-E}{2 \mu_{1}} \frac{2 x(y+h)}{\left(x^{2}+(y+h)^{2}\right)^{2}}, \quad u_{y}^{1}=\frac{-E}{2 \mu_{1}} \frac{(y+h)^{2}-x^{2}}{\left(x^{2}+(y+h)^{2}\right)^{2}} .
\end{aligned}
$$

Eq. (2.20) represent the solution for a double moment in the $x$-direction, applied at the image point of material 1. Setting $y=0$ in (2.20), and adding to (2.18), the continuity conditions at the interface are found to be satisfied if

$$
\begin{aligned}
& J_{1} h+2 E-J_{2} h=0, \\
& v J_{1} h+2 v E+J_{2} h=0, \\
& \left(A_{1}+\frac{f_{y}}{\pi\left(1+k_{1}\right)}-A_{2}\right) h^{2}+\left(I_{1}+J_{1}+I_{2}+J_{2}\right) h+E=0, \\
& \left(A_{1}+\frac{f_{y}}{\pi\left(1+k_{1}\right)}-A_{2}\right) h^{2}+\left(I_{1}+I_{2}\right) h-E=0, \\
& \left(v A_{1}+\frac{v f_{y}}{\pi\left(1+k_{1}\right)}-A_{2}\right) h^{2}+v\left(I_{1}+\frac{3-k_{1}}{4} J_{1}\right) h+v E+\left(I_{2}+\frac{3-k_{2}}{4} J_{2}\right)=0, \\
& \left(v A_{1}+\frac{v f_{y}}{\pi\left(1+k_{1}\right)}-A_{2}\right) h^{2}+v\left(I_{1}-\frac{1+k_{1}}{4} J_{1}\right) h-v E+\left(I_{2}-\frac{1+k_{2}}{4} J_{2}\right)=0 .
\end{aligned}
$$

We thus have eight equations, two of which are defined by (2.17), and the remaining six by (2.21), involving only five unknowns, namely $I_{1}, J_{1}, I_{2}, J_{2}$ and $E$. However, a careful examination reveals that these eight equations are not linearly independent. If these equations can be solved then the continuity conditions at the interface are guaranteed. The first two equations of (2.21) immediately furnish

$$
J_{2}=0, \quad E=\frac{-1}{2} J_{1} h
$$

Substituting (2.22) into the third and fourth equations of (2.21), we find that these two equations are identical, having the simplified form

$$
\left(A_{1}+\frac{f_{y}}{\pi\left(1+k_{1}\right)}-A_{2}\right) h^{2}+\left(I_{1}+I_{2}+\frac{J_{1}}{2}\right) h=0 .
$$

Similarly, the fifth and sixth equations of (2.21) are reduced to the single equation

$$
\left(v A_{1}+\frac{v f_{y}}{\pi\left(1+k_{1}\right)}-A_{2}\right) h^{2}+\left(v I_{1}+\frac{v\left(1-k_{1}\right)}{4} J_{1}+I_{2}\right) h=0 .
$$

Hence, the eight equations of (2.17) and (2.21) actually become four equations expressed by (2.17), (2.23) and (2.24) and only three equations are independent. The three nonzero coefficients $I_{1}, J_{1}$ and $I_{2}$ can be determined uniquely. Solving these equations, we obtain

$$
\begin{array}{ll}
I_{1}=\frac{(1-v)\left(1-k_{1}\right) f_{y} h}{\pi\left(1+v k_{1}\right)\left(1+k_{1}\right)}, & J_{1}=\frac{-4(1-v) f_{y} h}{\pi\left(1+v k_{1}\right)\left(1+k_{1}\right)} \\
E=\frac{2(1-v) f_{y} h^{2}}{\pi\left(1+v k_{1}\right)\left(1+k_{1}\right)}, & I_{2}=\frac{\left(1-k_{2}+v k_{1}-v\right) v f_{y} h}{\pi\left(1+v k_{1}\right)\left(v+k_{2}\right)} .
\end{array}
$$

Since all the unknown coefficients have been determined, Green's function for the bimaterial subjected to a concentrated force $f_{y}$ is derived. The structure of image singularities of materials 1 and 2 for the bimaterial will be discussed in what follows. 
The expressions (2.7), which represent the image singularities for material 1, include the solution for an infinite medium subjected to a concentrated vertical force $f_{y}^{\prime}$ and a dislocation with Burger's vector $b_{x}^{\prime}$, both acting at $x=0$ and $y=-h$. The magnitude of the concentrated force $f_{y}^{\prime}$ and the dislocation $b_{x}^{\prime}$ can be obtained from the relations

$$
\begin{aligned}
& \frac{f_{y}^{\prime}}{\pi\left(1+k_{1}\right)}+\frac{2 \mu_{1} b_{x}^{\prime}}{\pi\left(1+k_{1}\right)}=A_{1}, \\
& \frac{1-k_{1}}{4} \frac{f_{y}^{\prime}}{\pi\left(1+k_{1}\right)}+\frac{\mu_{1} b_{x}^{\prime}}{\pi\left(1+k_{1}\right)}=B_{1},
\end{aligned}
$$

where $A_{1}$ and $B_{1}$ have been determined and are expressed in (2.10). The results for $f_{y}^{\prime}$ and $b_{x}^{\prime}$ therefore become

$$
f_{y}^{\prime}=\frac{\left(k_{2}-v^{2} k_{1}\right) f_{y}}{\left(v+k_{2}\right)\left(1+v k_{1}\right)}, \quad b_{x}^{\prime}=\frac{f_{y}}{2 \mu_{1}} \frac{\left[k_{1}\left(v+k_{2}\right)-k_{2}\left(1+v k_{1}\right)\right]}{\left(v+k_{2}\right)\left(1+v k_{1}\right)} .
$$

Since the coefficients $I_{1}$ and $J_{1}$ in (2.13) have the relation $J_{1}=\left(-4 /\left(1-k_{1}\right)\right) I_{1}$ in view of (2.25), it follows that (2.13) is a solution for a double force without moment along the $x$-direction. The magnitude of the double force $P_{x}$ is given by

$$
I_{1}=\frac{P_{x}\left(k_{1}-1\right)}{2 \pi\left(1+k_{1}\right)} .
$$

Substituting from (2.25), the double force $P_{x}$ is found to be

$$
P_{x}=-\frac{2(1-v)}{\left(1+v k_{1}\right)} f_{y} h
$$

Finally, the magnitude of the double moment along the $x$-direction $M_{x}$ can be determined by the following equation

$$
E=\frac{M_{x}}{\pi}
$$

From (2.25), the result is

$$
M_{x}=\frac{2(1-v)}{\left(1+v k_{1}\right)\left(1+k_{1}\right)} f_{y} h^{2} .
$$

Hence, the stress function for all the image singularities and their magnitudes at the image point $y=-h$ of material 1, can be written in the following form

$$
\vec{\phi}^{i}=\vec{\phi}^{f_{y}^{\prime}}+\vec{\phi}^{b_{x}^{\prime}}+\vec{\phi}^{P_{x}}+\vec{\phi}^{M_{x}}
$$

Eq. (2.28) indicates that the image singularities for material 1 consist of a vertical concentrated force $f_{y}^{\prime}$, a dislocation with horizontal Burger's vector $b_{x}^{\prime}$, a double force without moment along the $x$-direction of magnitude $P_{x}$, and a double moment along the $x$-direction of magnitude $M_{x}$. It is noted that each image singularity has its own physical meaning, and is shown in Fig. 2, the magnitudes of these singularities being given by (2.27a), (2.27c) and (2.27e).

The image singularities for material 2 also include the solution for an infinite plane subjected to a concentrated force $f_{y}^{\prime}$ and dislocation $b_{x}^{\prime}$. Using (2.10), the magnitudes of the image concentrated force and dislocation of material 2 are obtained in a manner similar to that for material 1 , and the results are 


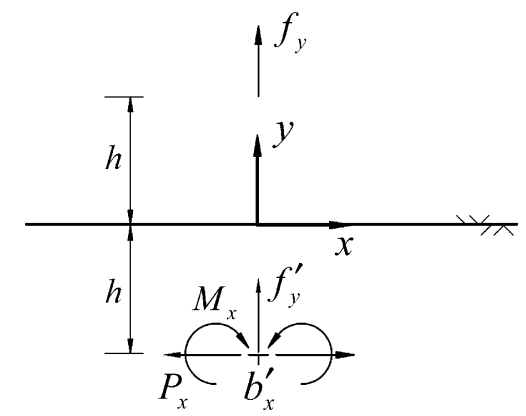

Fig. 2. Image singularities of material 1 for bimaterials subjected to a vertical concentrated force $f_{y}$ applied at material 1.

$$
f_{y}^{\prime}=\frac{v\left[\left(1+v k_{1}\right)+k_{1}\left(v+k_{2}\right)\right] f_{y}}{\left(v+k_{2}\right)\left(1+v k_{1}\right)}, \quad b_{x}^{\prime}=\frac{v\left[k_{2}\left(1+v k_{1}\right)-k_{1}\left(v+k_{2}\right)\right] f_{y}}{2 \mu_{2}\left(v+k_{2}\right)\left(1+v k_{1}\right)} .
$$

From the condition $J_{2}=0$, the expression in (2.14) represents the solution for the center of dilatation applied at $(0, h)$, the magnitude of the center of dilatation $D$ being

$$
D=-\frac{\left[\left(1+v k_{1}\right)-\left(v+k_{2}\right)\right]\left(1+k_{2}\right) v}{\left(1-k_{2}\right)\left(1+v k_{1}\right)\left(v+k_{2}\right)} f_{y} h .
$$

Only three image singularities exist for material 2, and are expressed by the stress function

$$
\overrightarrow{\phi^{i}}=\vec{\phi}^{f_{y}^{\prime}}+\vec{\phi}^{b_{x}^{\prime}}+\vec{\phi}^{D}
$$

The three image singularities of material 2 consist of a vertical concentrated force $f_{y}^{\prime}$, a dislocation with horizontal Burger's vector $b_{x}^{\prime}$, and a center of dilatation $D$, as shown in Fig. 3 .

The full field stress distributions due to point loads acting in an isotropic bimaterial have been obtained by some authors. For a verification of the solution presented in this paper, we compare the normal stress $\sigma_{y y}$ according to this investigation with that obtained by Ma and Huang (1996). The full field two-dimensional plane strain solution for the normal stresses $\sigma_{y y}$ for the bimaterials are (Ma and Huang (1996), Eqs. (75) and (76)).

$$
\begin{aligned}
\sigma_{y y}^{1}=\frac{f_{y}}{2 \pi}\{ & -\frac{(y-h)}{x^{2}+(y-h)^{2}}+\frac{(y-h)\left(x^{2}-(y-h)^{2}\right)}{2\left(1-v_{1}\right)\left(x^{2}+(y-h)^{2}\right)^{2}} \\
& +\frac{2(1-v)}{\left(1-v_{1}\right)\left(1+v\left(3-4 v_{1}\right)\right)} \frac{y h(y+h)\left(3 x^{2}-(y+h)^{2}\right)}{\left(x^{2}+(y+h)^{2}\right)^{3}} \\
& +\frac{1-v}{2\left(1-v_{1}\right)\left(1+v\left(3-4 v_{1}\right)\right)} \frac{\left(\left(3-4 v_{1}\right) y+h\right)\left(x^{2}-(y+h)^{2}\right)}{\left(x^{2}+(y+h)^{2}\right)^{2}} \\
& \left.-\frac{3\left(1-v^{2}\right)+4\left(v_{1} v^{2}-v_{2}\right)}{\left(v+3-4 v_{2}\right)\left(1+v\left(3-4 v_{1}\right)\right)} \frac{y+h}{x^{2}+(y+h)^{2}}\right\},
\end{aligned}
$$




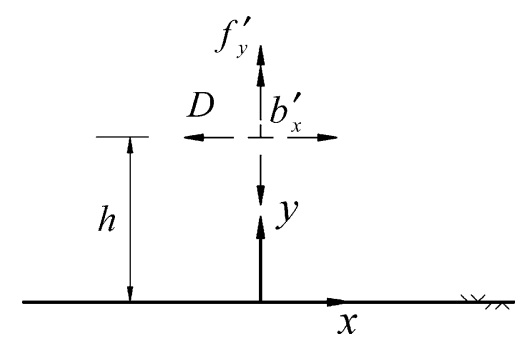

Fig. 3. Image singularities of material 2 for bimaterials subjected to a vertical concentrated force $f_{y}$ applied at material 1.

for material 1 and

$$
\begin{aligned}
\sigma_{y y}^{2}=\frac{f_{y}}{2 \pi} & \left\{\frac{2\left[v^{2}\left(3-4 v_{1}\right)+v\left(5-6\left(v_{1}+v_{2}\right)+8 v_{1} v_{2}\right)\right]}{\left(v+3-4 v_{2}\right)\left(1+v\left(3-4 v_{1}\right)\right)} \frac{y-h}{x^{2}+(y-h)^{2}}-\left[\frac{2 v y}{v+3-4 v_{2}}+\frac{2 v h}{1+v\left(3-4 v_{1}\right)}\right]\right. \\
& \left.\times \frac{x^{2}-(y-h)^{2}}{\left(x^{2}+(y-h)^{2}\right)^{2}}\right\}
\end{aligned}
$$

for material 2. After some algebraic manipulation, the solutions represented by (2.31a) and (2.31b) can be rearranged as follows:

$$
\begin{aligned}
& \sigma_{y y}^{1}=\frac{-f_{y}}{2 \pi}\left[\frac{y-h}{x^{2}+(y-h)^{2}}-\frac{2}{1+k_{1}} \frac{(y-h)\left(x^{2}-(y-h)^{2}\right)}{\left(x^{2}+(y-h)^{2}\right)^{2}}\right] \quad \text { (Applied force) } \\
& -\frac{\left(k_{2}-v^{2} k_{1}\right) f_{y}}{2 \pi\left(v+k_{2}\right)\left(1+v k_{1}\right)}\left[\frac{y+h}{x^{2}+(y+h)^{2}}-\frac{2}{1+k_{1}} \frac{(y+h)\left(x^{2}-(y+h)^{2}\right)}{\left(x^{2}+(y+h)^{2}\right)^{2}}\right] \\
& +\frac{\left(v k_{1}+k_{1} k_{2}-k_{2}-v k_{1} k_{2}\right) f_{y}}{\pi\left(1+k_{1}\right)\left(v+k_{2}\right)\left(1+v k_{1}\right)}\left[\frac{(y+h)\left(x^{2}-(y+h)^{2}\right)}{\left(x^{2}+(y+h)^{2}\right)^{2}}\right] \quad \text { (Image dislocation) } \\
& -\frac{(1-v) f_{y} h}{\pi\left(1+k_{1}\right)\left(1+v k_{1}\right)}\left[\left(1-k_{1}\right) \frac{(y+h)^{2}-x^{2}}{\left(x^{2}+(y+h)^{2}\right)^{2}}+4 \frac{(y+h)^{2}\left((y+h)^{2}-3 x^{2}\right)}{\left(x^{2}+(y+h)^{2}\right)^{3}}\right] \\
& \text { (Image double force without moment) } \\
& +\frac{4(1-v) f_{y} h^{2}}{\pi\left(1+k_{1}\right)\left(1+v k_{1}\right)}\left[\frac{(y+h)\left((y+h)^{2}-3 x^{2}\right)}{\left(x^{2}+(y+h)^{2}\right)^{3}}\right] \quad \text { (Image double moment) }
\end{aligned}
$$




$$
\begin{aligned}
\sigma_{y y}^{2}= & \frac{-v\left(1+2 v k_{1}+k_{1} k_{2}\right) f_{y}}{2 \pi\left(v+k_{2}\right)\left(1+v k_{1}\right)}\left[\frac{y-h}{x^{2}+(y-h)^{2}}+\frac{2}{1+k_{2}} \frac{(y-h)\left((y-h)^{2}-x^{2}\right)}{\left(x^{2}+(y-h)^{2}\right)^{2}}\right] \quad(\text { Imag } \\
& +\frac{v\left(v k_{1} k_{2}-v k_{1}+k_{2}-k_{1} k_{2}\right) f_{y}}{\pi\left(1+k_{2}\right)\left(v+k_{2}\right)\left(1+v k_{1}\right)}\left[\frac{(y-h)\left(x^{2}-(y-h)^{2}\right)}{\left(x^{2}+(y-h)^{2}\right)^{2}}\right] \quad \text { (Image dislocation) } \\
& -\frac{v\left(1-k_{2}+v k_{1}-v\right) f_{y} h}{\pi\left(v+k_{2}\right)\left(1+v k_{1}\right)}\left[\frac{(y-h)^{2}-x^{2}}{\left(x^{2}+(y-h)^{2}\right)^{2}}\right] \quad \text { (Image center of dilatation). }
\end{aligned}
$$

It is interesting to note that the complicated Green's function given by (2.31a) and (2.31b) for a bimaterial system subjected to a concentrated vertical force $f_{y}$ in material 1 can be represented by a combination of several Green's functions for a homogeneous infinite medium. For material 1, the complete solution given by (2.32a)-(2.32e) consist of two parts. The first term (i.e. (2.32a)) represents Green's function for a concentrated force $f_{y}$ applied at $(0, h)$ in an infinite medium, which the other terms (i.e. (2.32b)-(2.32e)) represent four Green's functions for the infinite medium with loads applied at $(0,-h)$, which is the image point of material 1. The solution for material 2 can also be expressed as a combination of three Green's functions for an infinite medium by applying loads at $(0, h)$, which is the image point of material 2 .

Recalling that $\sigma_{y y}=\phi_{y, x}$, we differentiate the stress functions $\phi_{y}$ of the singularities obtained previously, and obtain the result for the stress $\sigma_{y y}$ as

$$
\sigma_{y y}^{1}=\left(\phi_{y, x}^{\mathrm{o}}\right)_{\text {applied singularity }}^{1}+\left(\phi_{y, x}^{f_{y}^{\prime}}+\phi_{y, x}^{b_{x}^{\prime}}+\phi_{y, x}^{P_{x}}+\phi_{y, x}^{M_{x}}\right)_{\text {image singularity }}^{1},
$$

for material 1 , and

$$
\sigma_{y y}^{2}=\left(\phi_{y, x}^{f_{y}^{\prime}}+\phi_{y, x}^{b_{x}^{\prime}}+\phi_{y, x}^{D}\right)_{\text {image singularity }}^{2},
$$

for material 2. The five terms in (2.34) are exactly the same as the terms in (2.32a)-(2.32e), while the three terms in (2.35) are exactly the same as those in (2.33a)-(2.33c).

\subsection{Remarks and limiting cases}

(1) Let the applied vertical force $f_{y}$ approaches the interface, i.e. $h \rightarrow 0$. Then the values of $I_{1}, I_{2}, J_{1}$ and $E$ in (2.25) will be equal to zero, and Green's functions of a bimaterial subjected to a vertical concentrated force $f_{y}$ applied at the interface is reduced to

$$
\begin{aligned}
\phi_{x}^{1} & =\frac{-f_{y}}{2 \pi} \frac{v\left(k_{1} k_{2}-1\right) \ln r-2\left(k_{2}+v\right) \sin ^{2} \theta}{\left(v+k_{2}\right)\left(1+k_{1} v\right)} \\
\phi_{y}^{1} & =\frac{-f_{y}}{2 \pi} \frac{\left(k_{2}+v\right) \sin 2 \theta-\left(v\left(1+k_{1} k_{2}\right)+2 k_{2}\right) \theta}{\left(v+k_{2}\right)\left(1+k_{1} v\right)} \\
u_{x}^{1} & =\frac{f_{y}}{4 \pi \mu_{1}} \frac{\left(k_{2}+v\right) \sin 2 \theta+\left(k_{1}\left(k_{2}+v\right)-k_{2}\left(1+k_{1} v\right)\right) \theta}{\left(v+k_{2}\right)\left(1+k_{1} v\right)}, \\
u_{y}^{1} & =\frac{f_{y}}{4 \pi \mu_{1}} \frac{2\left(k_{2}+v\right) \sin ^{2} \theta-\left[k_{1}\left(k_{2}+v\right)+k_{2}\left(1+k_{1} v\right)\right] \ln r}{\left(v+k_{2}\right)\left(1+k_{1} v\right)},
\end{aligned}
$$


for material 1 , and

$$
\begin{aligned}
\phi_{x}^{2} & =\frac{-f_{y}}{2 \pi} \frac{v\left(k_{1} k_{2}-1\right) \ln r-2 v\left(1+k_{1} v\right) \sin ^{2} \theta}{\left(v+k_{2}\right)\left(1+k_{1} v\right)}, \\
\phi_{y}^{2} & =\frac{-f_{y}}{2 \pi} \frac{v\left(1+k_{1} v\right) \sin 2 \theta-\left[v\left(1+k_{1} k_{2}\right)+2 k_{1} v^{2}\right] \theta}{\left(v+k_{2}\right)\left(1+k_{1} v\right)}, \\
u_{x}^{2} & =\frac{f_{y}}{4 \pi \mu_{2}} \frac{\left(1+k_{1} v\right) \sin 2 \theta-\left[k_{1}\left(k_{2}+v\right)-k_{2}\left(1+k_{1} v\right)\right] \theta}{\left(v+k_{2}\right)\left(1+k_{1} v\right)}, \\
u_{y}^{2} & =\frac{f_{y}}{4 \pi \mu_{2}} \frac{2\left(1+k_{1} v\right) \sin ^{2} \theta-\left[k_{1}\left(k_{2}+v\right)+k_{2}\left(1+k_{1} v\right)\right] \ln r}{\left(v+k_{2}\right)\left(1+k_{1} v\right)},
\end{aligned}
$$

for material 2, where $r=\left(x^{2}+y^{2}\right)^{1 / 2}$, and $\theta=\tan ^{-1}(y / x)$. The solutions presented in (2.36) and (2.37) are the same as those obtained by Ting (1996).

(2) The magnitudes of image singularities with stress singularity of order $1 / r^{2}$ (double force without moment and center of dilatation) and of order $1 / r^{3}$ (double moment) are proportional to $h$ and $h^{2}$ respectively. Hence, when the applied force $f_{y}$ approaches the interface (i.e. $h \rightarrow 0$ ) these higher order singularities will disappear. Only the concentrated force $f_{y}$ and dislocation $b_{x}$ with stress singularity $1 / r$ will exist at the image point. Note that when the applied force approaches the interface, the object point and the image point coincide. This indicates that the solution for a bimaterial subjected to a vertical concentrated force applied at the interface, $x=0$ and $y=0$, is equal to the sum of the solutions for an infinite homogeneous medium corresponding to vertical concentrated forces and dislocations applied at the point $x=0$ and $y=0$.

(3) It is interesting to note that the highest order of stress singularity at the image points is $1 / r^{3}$ (double moment) for material 1 , and $1 / r^{2}$ (center of dilatation) for material 2.

\begin{tabular}{|c|c|c|c|c|c|}
\hline Material & $\begin{array}{l}\text { Magnitude of image } \\
\text { singularity }\end{array}$ & Bimaterials & $\begin{array}{l}\text { Half-plane with free } \\
\text { boundary }\end{array}$ & $\begin{array}{l}\text { Half-plane with rig- } \\
\text { idly fixed boundary }\end{array}$ & $\begin{array}{l}\text { Infinite } \\
\text { plane }\end{array}$ \\
\hline \multirow[t]{4}{*}{1} & $f_{y}^{\prime}$ & $\frac{k_{2}-v^{2} k_{1}}{\left(v+k_{2}\right)\left(1+v k_{1}\right)} f_{y}$ & $f_{y}$ & $-f_{y}$ & 0 \\
\hline & $b_{x}^{\prime}$ & $\frac{\left[k_{1}\left(v+k_{2}\right)-k_{2}\left(1+v k_{1}\right)\right] f_{y}}{2 \mu_{1}\left(v+k_{2}\right)\left(1+v k_{1}\right)}$ & $\frac{\left(k_{1}-1\right) f_{y}}{2 \mu_{1}}$ & 0 & 0 \\
\hline & $P_{x}$ & $-\frac{2(1-v) f_{y} h}{\left(1+v k_{1}\right)}$ & $-2 f_{y} h$ & $\frac{2 f_{y} h}{k_{1}}$ & 0 \\
\hline & $M_{x}$ & $\frac{2(1-v) f_{y} h^{2}}{\left(1+v k_{1}\right)\left(1+k_{1}\right)}$ & $\frac{2 f_{y} h^{2}}{1+k_{1}}$ & $-\frac{2 f_{y} h^{2}}{\left(1+k_{1}\right) k_{1}}$ & 0 \\
\hline \multirow[t]{3}{*}{2} & $f_{y}^{\prime}$ & $\frac{v\left[\left(1+v k_{1}\right)+k_{1}\left(v+k_{2}\right)\right] f_{y}}{\left(v+k_{2}\right)\left(1+v k_{1}\right)}$ & 0 & $2 f_{y}$ & $f_{y}$ \\
\hline & $b_{x}^{\prime}$ & $\frac{v\left[k_{2}\left(1+v k_{1}\right)-k_{1}\left(v+k_{2}\right)\right] f_{y}}{2 \mu_{2}\left(v+k_{2}\right)\left(1+v k_{1}\right)}$ & $\frac{\left(1-k_{1}\right) f_{y}}{2 \mu_{1}}$ & 0 & 0 \\
\hline & $D$ & $\frac{v\left(1+k_{2}\right)\left[v+k_{2}-1-v k_{1}\right] f_{y} h}{\left(1-k_{2}\right)\left(1+v k_{1}\right)\left(v+k_{2}\right)}$ & 0 & $\frac{\left(1+k_{2}\right)\left(1-k_{1}\right) f_{y} h}{\left(1-k_{2}\right) k_{1}}$ & 0 \\
\hline
\end{tabular}

Table 1

The structures and magnitudes of image singularities for bimaterials and special cases of half-plane and infinite plane subjected to vertical concentrated force $f_{y}$ 
(4) The special case of a half-plane with free (or rigidly fixed) boundary, and that of an infinite plane, can be obtained by letting $\mu_{2} \rightarrow 0$ (or $\mu_{2} \rightarrow \infty$ ), and $\mu_{2}=\mu_{1}$, respectively, the corresponding results being summarized in Table 1 . The image singularities for a half-plane have been derived and discussed in detail by Ma and Lin (2001).

\section{Image singularities of bimaterials subjected to other types of force}

The image singularities for bimaterial system subjected to a horizontal concentrated force or dislocations will be discussed in this section following the similar procedure presented in the previous section. Leaving out the algebraic details, only the final results will be presented.

\subsection{Image singularities of bimaterials subjected to a horizontal concentrated force $f_{x}$}

Polar coordinate and matrix forms will be used in this section to present the solutions. The definitions of the following new variables are illustrated in Fig. 4

$$
\begin{aligned}
& r_{1}=\sqrt{x^{2}+(y-h)^{2}}, \quad r_{2}=\sqrt{x^{2}+(y+h)^{2}}, \quad \theta_{1}=\tan ^{-1} \frac{y-h}{x} \\
& \theta_{2}=\tan ^{-1} \frac{y+h}{x}, \quad \mathbf{m}\left(\theta_{j}\right)=\left[\begin{array}{lll}
\cos \theta_{j} & \sin \theta_{j}
\end{array}\right]^{\mathrm{T}}, \quad \text { and } \quad \mathbf{n}\left(\theta_{j}\right)=\left[\begin{array}{ll}
-\sin \theta_{j} & \cos \theta_{j}
\end{array}\right]^{\mathrm{T}} .
\end{aligned}
$$

The solutions to the stress functions and displacements in an infinite plane subjected to a horizontal concentrated force $f_{x}$ at the object point $(0, h)$ are

$$
\begin{aligned}
& \vec{\phi}=\left\{\begin{array}{l}
\phi_{x} \\
\phi_{y}
\end{array}\right\}=\frac{f_{x}}{\pi\left(1+k_{1}\right)}\left\{\sin \theta_{1} \mathbf{m}\left(\theta_{1}\right)+\left[\begin{array}{c}
\frac{1+k_{1}}{2} \theta_{1} \\
-\frac{1-k_{1}}{2} \ln r_{1}
\end{array}\right]\right\}, \\
& \vec{u}=\left\{\begin{array}{l}
u_{x} \\
u_{y}
\end{array}\right\}=\frac{f_{x}}{2 \pi \mu_{1}\left(1+k_{1}\right)}\left\{\sin \theta_{1} \mathbf{n}\left(\theta_{1}\right)-\left[\begin{array}{c}
k_{1} \ln r_{1} \\
0
\end{array}\right]\right\} .
\end{aligned}
$$

The components of image singularities for the bimaterial can be obtained by a procedure similar to that used in Section 2.2. The image singularities of material 1 at the image point $(0,-h)$ include a horizontal concentrated force $f_{x}^{\prime}$, a dislocation with vertical Burger's vector $b_{y}^{\prime}$, a double force with moment along the $x$-direction equal to $C_{x}$, and a double moment along the $y$-direction equal to $M_{y}$.

The resultant solution for the image singularities can be denoted by the expression

$$
\overrightarrow{\phi^{i}}=\vec{\phi}^{f_{x}^{\prime}}+\vec{\phi}^{b_{y}^{\prime}}+\vec{\phi}^{C_{x}}+\vec{\phi}^{M_{y}}
$$

for the stress function, and by a similar one for the displacement.

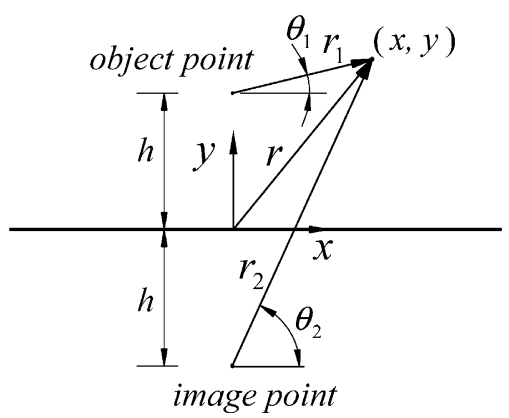

Fig. 4. The Geometric configuration of the definition for $r_{1}, \theta_{1}, r_{2}$ and $\theta_{2}$. 
The solutions and magnitudes for the various image singularities of material 1 are expressed as follows

$$
\begin{aligned}
\vec{\phi}^{f_{x}^{\prime}} & =\frac{f_{x}^{\prime}}{\pi\left(1+k_{1}\right)}\left\{\sin \theta_{2} \mathbf{m}\left(\theta_{2}\right)+\left[\begin{array}{c}
\frac{1+k_{1}}{2} \theta_{2} \\
-\frac{1-k_{1}}{2} \ln r_{2}
\end{array}\right]\right\}, \\
\vec{u}^{f_{x}^{\prime}} & =\frac{f_{x}^{\prime}}{2 \pi \mu_{1}\left(1+k_{1}\right)}\left\{\sin \theta_{2} \mathbf{n}\left(\theta_{2}\right)-\left[\begin{array}{c}
k_{1} \ln r_{2} \\
0
\end{array}\right]\right\}, \quad f_{x}^{\prime}=\frac{\left(k_{2}-v^{2} k_{1}\right) f_{x}}{\left(v+k_{2}\right)\left(1+v k_{1}\right)} . \\
\vec{\phi}^{b_{y}^{\prime}} & =\frac{-2 \mu_{1} b_{y}^{\prime}}{\pi\left(1+k_{1}\right)}\left\{\sin \theta_{2} \mathbf{m}\left(\theta_{2}\right)-\left[\begin{array}{c}
0 \\
\ln r_{2}
\end{array}\right]\right\}, \\
\vec{u}^{b_{y}^{\prime}} & =\frac{-b_{y}^{\prime}}{\pi\left(1+k_{1}\right)}\left\{\sin \theta_{2} \mathbf{n}\left(\theta_{2}\right)+\left[\begin{array}{c}
\frac{1-k_{1}}{2} \ln r_{2} \\
-\frac{1+k_{1}}{2} \theta_{2}
\end{array}\right]\right\}, \quad b_{y}^{\prime}=\frac{f_{x}}{2 \mu_{1}} \frac{\left[k_{2}\left(1+v k_{1}\right)-k_{1}\left(v+k_{2}\right)\right]}{\left(v+k_{2}\right)\left(1+v k_{1}\right)} . \\
\vec{\phi}^{C_{x}} & =\frac{-C_{x}}{\pi\left(1+k_{1}\right) r_{2}}\left(\frac{1+k_{1}}{2}-\cos 2 \theta_{2}\right) \mathbf{n}\left(\theta_{2}\right), \\
\vec{u}^{C_{x}} & =\frac{-C_{x}}{2 \pi \mu_{1}\left(1+k_{1}\right) r_{2}}\left\{\left[\begin{array}{cc}
1 & 0 \\
0 & k_{1}
\end{array}\right] \mathbf{n}\left(\theta_{2}\right)+\sin 2 \theta_{2} \mathbf{m}\left(\theta_{2}\right)\right\}, \quad C_{x}=\frac{2(1-v)}{\left(1+v k_{1}\right)} f_{x} h . \\
\vec{\phi}^{M_{y}} & =\frac{M_{y}}{\pi r_{2}^{2}}\left(\sin \theta_{2} \mathbf{m}\left(\theta_{2}\right)-\cos \theta_{2} \mathbf{n}\left(\theta_{2}\right)\right), \quad \vec{u}^{M_{y}}=\frac{M_{y}}{2 \pi \mu_{1} r_{2}^{2}}\left(\cos \theta_{2} \mathbf{m}\left(\theta_{2}\right)+\sin \theta_{2} \mathbf{n}\left(\theta_{2}\right)\right), \\
M_{y} & =\frac{2(1-v)}{\left(1+k_{1}\right)\left(1+v k_{1}\right)} f_{x} h^{2} .
\end{aligned}
$$

For material 2, the image singularities at the image point $(0, h)$ consist of a horizontal concentrated force $f_{x}^{\prime}$, a dislocation with vertical Burger's vector $b_{y}^{\prime}$, and a concentrated moment $M$, the result being

$$
\overrightarrow{\phi^{i}}=\vec{\phi}^{f_{x}^{\prime}}+\vec{\phi}^{b_{y}^{\prime}}+\vec{\phi}^{M}
$$

The solutions and magnitudes of the individual image singularities are

$$
\begin{aligned}
& \vec{\phi}^{f_{x}^{\prime}}=\frac{f_{x}^{\prime}}{\pi\left(1+k_{2}\right)}\left\{\sin \theta_{1} \mathbf{m}\left(\theta_{1}\right)+\left[\begin{array}{c}
\frac{1+k_{2}}{2} \theta_{1} \\
-\frac{1-k_{2}}{2} \ln r_{1}
\end{array}\right]\right\}, \\
& \vec{u}^{f_{x}^{\prime}}=\frac{f_{x}^{\prime}}{2 \pi \mu_{2}\left(1+k_{2}\right)}\left\{\sin \theta_{1} \mathbf{n}\left(\theta_{1}\right)-\left[\begin{array}{c}
k_{2} \ln r_{1} \\
0
\end{array}\right]\right\}, \quad f_{x}^{\prime}=\frac{v\left[\left(1+v k_{1}\right)+k_{1}\left(v+k_{2}\right)\right]}{\left(v+k_{2}\right)\left(1+v k_{1}\right)} f_{x} . \\
& \vec{\phi}^{b_{y}^{\prime}}=\frac{-2 \mu_{2} b_{y}^{\prime}}{\pi\left(1+k_{2}\right)}\left\{\sin \theta_{1} \mathbf{m}\left(\theta_{1}\right)-\left[\begin{array}{c}
0 \\
\ln r_{1}
\end{array}\right]\right\}, \\
& \vec{u}^{b_{y}^{\prime}}=\frac{-b_{y}^{\prime}}{\pi\left(1+k_{2}\right)}\left\{\sin \theta_{1} \mathbf{n}\left(\theta_{1}\right)+\left[\begin{array}{c}
\frac{1-k_{2}}{2} \ln r_{1} \\
-\frac{1+k_{2}}{2} \theta_{1}
\end{array}\right]\right\}, \quad b_{y}^{\prime}=\frac{-f_{x}}{2 \mu_{1}} \frac{\left[k_{2}\left(1+v k_{1}\right)-k_{1}\left(v+k_{2}\right)\right]}{\left(v+k_{2}\right)\left(1+v k_{1}\right)}, \\
& \vec{\phi}^{M}=\frac{M}{\pi r_{1}} \mathbf{m}\left(\theta_{1}\right), \quad \vec{u}^{M}=\frac{M}{2 \pi \mu_{2} r_{1}} \mathbf{n}\left(\theta_{1}\right), \quad M=\frac{v\left[\left(1+v k_{1}\right)-\left(v+k_{2}\right)\right]}{\left(v+k_{2}\right)\left(1+v k_{1}\right)} f_{x} h .
\end{aligned}
$$




\subsection{Image singularities of bimaterials subjected to a dislocation with Burger's vector $b_{x}$}

The solutions to the stress functions and displacements for a dislocation with magnitude $b_{x}$ applied at the object point $(0, h)$ of an infinite plane for material 1 are

$$
\begin{aligned}
& \vec{\phi}=\frac{-2 \mu_{1} b_{x}}{\pi\left(1+k_{1}\right)}\left\{\sin \theta_{1} \mathbf{n}\left(\theta_{1}\right)-\left[\begin{array}{c}
\ln r_{1} \\
0
\end{array}\right]\right\}, \\
& \vec{u}=\frac{b_{x}}{\pi\left(1+k_{1}\right)}\left\{\sin \theta_{1} \mathbf{m}\left(\theta_{1}\right)+\left[\begin{array}{c}
\frac{1+k_{1}}{2} \theta_{1} \\
\frac{1-k_{1}}{2} \ln r_{1}
\end{array}\right]\right\} .
\end{aligned}
$$

The structure of image singularities at the image point $(0,-h)$ of material 1 is

$$
\vec{\phi}^{i}=\vec{\phi}_{y}^{f_{y}^{\prime}}+\vec{\phi}^{b_{x}^{\prime}}+\vec{\phi}^{\gamma_{x}}+\vec{\phi}^{x_{y}}
$$

Eq. (3.13) indicates that the image singularities include a vertical concentrated force $f_{y}^{\prime}$, a dislocation with horizontal Burger's vector $b_{x}^{\prime}$, a center of extension along the $x$-direction equal to $\gamma_{x}$, and a double center of expansion along $y$-direction equal to $\chi_{y}$ (see Fig. 5). The solutions and magnitudes of these image singularities are

$$
\begin{aligned}
& \vec{\phi}^{f_{y}^{\prime}}=\frac{-f_{y}^{\prime}}{\pi\left(1+k_{1}\right)}\left\{\sin \theta_{2} \mathbf{n}\left(\theta_{2}\right)+\left[\begin{array}{c}
-\frac{1-k_{1}}{2} \ln r_{2} \\
\frac{1+k_{1}}{2} \theta_{2}
\end{array}\right]\right\}, \\
& \vec{u}_{y}^{f_{y}^{\prime}}=\frac{f_{y}^{\prime}}{2 \pi \mu_{1}\left(1+k_{1}\right)}\left\{\sin \theta_{2} \mathbf{m}\left(\theta_{2}\right)-\left[\begin{array}{c}
0 \\
-k_{1} \ln r_{2}
\end{array}\right]\right\}, \quad f_{y}^{\prime}=\frac{2 \mu_{1} b_{x} v\left[\left(v+k_{2}\right)-\left(1+v k_{1}\right)\right]}{\left(v+k_{2}\right)\left(1+v k_{1}\right)} . \\
& \vec{\phi}^{b_{x}^{\prime}}=\frac{-2 \mu_{1} b_{x}^{\prime}}{\pi\left(1+k_{1}\right)}\left\{\sin \theta_{2} \mathbf{n}\left(\theta_{2}\right)-\left[\begin{array}{c}
\ln r_{2} \\
0
\end{array}\right]\right\}, \\
& \vec{u}^{b_{x}^{\prime}}=\frac{b_{x}^{\prime}}{\pi\left(1+k_{1}\right)}\left\{\sin \theta_{2} \mathbf{m}\left(\theta_{2}\right)+\left[\begin{array}{c}
\frac{1+k_{1}}{2} \theta_{2} \\
\frac{1-k_{1}}{2} \ln r_{2}
\end{array}\right]\right\}, \quad b_{x}^{\prime}=\frac{\left(v^{2} k_{1}-k_{2}\right)}{\left(v+k_{2}\right)\left(1+v k_{1}\right)} b_{x} .
\end{aligned}
$$

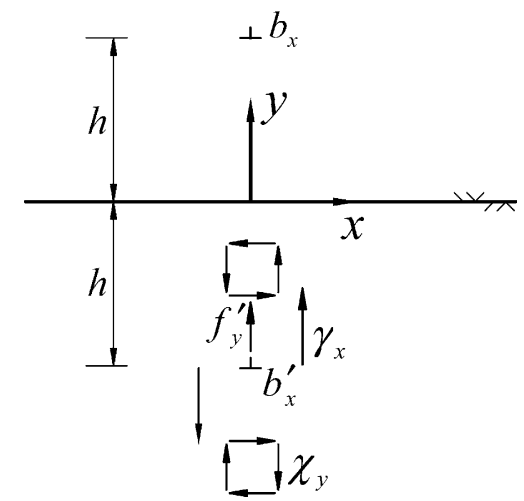

Fig. 5. Image singularities of material 1 for bimaterials subjected to a dislocation with Burger's vector $b_{x}$ applied at material 1 . 


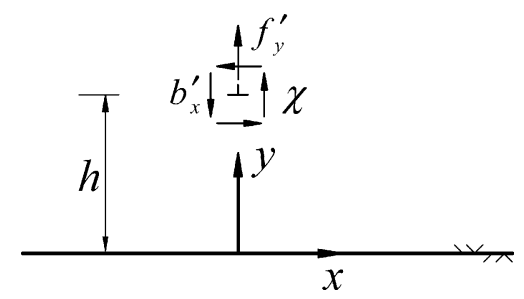

Fig. 6. Image singularities of material 2 for bimaterial subjected to a dislocation with Burger's vector $b_{x}$ applied at material 1 .

$$
\begin{aligned}
\vec{\phi}^{\gamma_{x}} & =\frac{-2 \mu_{1} \gamma_{x}}{\pi\left(1+k_{1}\right) r_{2}}\left\{\mathbf{n}\left(\theta_{2}\right)+\sin 2 \theta_{2} \mathbf{m}\left(\theta_{2}\right)\right\}, \\
\vec{u}^{\gamma_{x}} & =\frac{\gamma_{x}}{2 \pi\left(1+k_{1}\right) r_{2}}\left\{\left[\begin{array}{cc}
1-k_{1} & 0 \\
0 & 3+k_{1}
\end{array}\right] \mathbf{m}\left(\theta_{2}\right)-2 \sin \theta_{2} \mathbf{n}\left(\theta_{2}\right)\right\}, \quad \gamma_{x}=\frac{2(1-v)}{1+v k_{1}} b_{x} h . \\
\vec{\phi}^{\chi_{y}} & =\frac{-4 \mu \chi_{y}}{\pi\left(1+k_{1}\right) r_{2}^{2}}\left(\cos \theta_{2} \mathbf{m}\left(\theta_{2}\right)+\sin \theta_{2} \mathbf{n}\left(\theta_{2}\right)\right), \\
\vec{u}^{\chi_{y}} & =\frac{2 \chi_{y}}{\pi\left(1+k_{1}\right) r_{2}^{2}}\left(\sin \theta_{2} \mathbf{m}\left(\theta_{2}\right)-\cos \theta_{2} \mathbf{n}\left(\theta_{2}\right)\right), \quad \chi_{y}=\frac{v-1}{1+v k_{1}} b_{x} h^{2} .
\end{aligned}
$$

For material 2, the image singularities consist of a vertical concentrated force $f_{y}^{\prime}$, a dislocation with horizontal Burger's vector $b_{x}^{\prime}$, and a center of expansion $\chi$ (see Fig. 6). The results are expressed as

$$
\overrightarrow{\phi^{i}}=\vec{\phi}^{f_{y}^{\prime}}+\vec{\phi}^{b_{x}^{\prime}}+\vec{\phi}^{x}
$$

where the solutions and magnitudes are

$$
\begin{aligned}
& \vec{\phi}^{f_{y}^{\prime}}=\frac{-f_{y}^{\prime}}{\pi\left(1+k_{2}\right)}\left\{\sin \theta_{1} \mathbf{n}\left(\theta_{1}\right)+\left[\begin{array}{c}
-\frac{1-k_{2}}{2} \ln r_{1} \\
\frac{1+k_{2}}{2} \theta_{1}
\end{array}\right]\right\}, \\
& \vec{u}_{y}^{f_{y}^{\prime}}=\frac{f_{y}^{\prime}}{2 \pi \mu_{2}\left(1+k_{2}\right)}\left\{\sin \theta_{1} \mathbf{m}\left(\theta_{1}\right)+\left[\begin{array}{c}
0 \\
k_{2} \ln r_{1}
\end{array}\right]\right\}, \quad f_{y}^{\prime}=\frac{2 \mu_{1} b_{x} v\left[\left(1+v k_{1}\right)-\left(v+k_{2}\right)\right]}{\left(v+k_{2}\right)\left(1+v k_{1}\right)} . \\
& \vec{\phi}^{b_{x}^{\prime}}=\frac{-2 \mu_{2} b_{x}^{\prime}}{\pi\left(1+k_{2}\right)}\left\{\sin \theta_{1} \mathbf{n}\left(\theta_{1}\right)-\left[\begin{array}{c}
\ln r_{1} \\
0
\end{array}\right]\right\}, \\
& \vec{\phi}^{b_{x}^{\prime}}=\frac{b_{x}^{\prime}}{\pi\left(1+k_{2}\right)}\left\{\sin \theta_{1} \mathbf{m}\left(\theta_{1}\right)+\left[\begin{array}{c}
\frac{1+k_{2}}{2} \theta_{1} \\
\frac{1-k_{2}}{2} \ln r_{1}
\end{array}\right]\right\}, \quad b_{x}^{\prime}=\frac{\left[\left(v+k_{2}\right)+k_{2}\left(1+v k_{1}\right)\right]}{\left(v+k_{2}\right)\left(1+v k_{1}\right)} b_{x} . \\
& \vec{\phi}^{\chi}=\frac{-4 \mu_{2} \chi}{\pi\left(1+k_{2}\right)} \mathbf{n}\left(\theta_{1}\right), \quad \vec{u}^{\chi}=\frac{2 \chi}{\pi\left(1+k_{2}\right)} \mathbf{m}\left(\theta_{1}\right), \quad \chi=\frac{\left(1+k_{2}\right)\left[\left(1+v k_{1}\right)-\left(v+k_{2}\right)\right]}{2\left(v+k_{2}\right)\left(1+v k_{1}\right)} b_{x} h .
\end{aligned}
$$

\subsection{Image singularities of bimaterials subjected to a dislocation with Burger's vector $b_{y}$}

The solutions to the stress functions and displacements for a dislocation with magnitude $b_{y}$ applied at the object point $(0, h)$ of material 1 on an infinite plane are 


$$
\vec{\phi}=\frac{-2 \mu_{1} b_{y}}{\pi\left(1+k_{1}\right)}\left\{\sin \theta_{1} \mathbf{m}\left(\theta_{1}\right)-\left[\begin{array}{c}
0 \\
\ln r_{1}
\end{array}\right]\right\}, \quad \vec{u}=\frac{-b_{y}}{\pi\left(1+k_{1}\right)}\left\{\sin \theta_{1} \mathbf{n}\left(\theta_{1}\right)+\left[\begin{array}{c}
\frac{1-k_{1}}{2} \ln r_{1} \\
-\frac{1+k_{1}}{2} \theta_{1}
\end{array}\right]\right\} .
$$

The structure of image singularities at the image point $(0,-h)$ of material 1 is

$$
\vec{\phi}^{i}=\vec{\phi}^{f_{x}^{\prime}}+\vec{\phi}^{b_{y}^{\prime}}+\vec{\phi}^{\kappa_{x}}+\vec{\phi}^{\chi_{x}}
$$

The image singularities for this case include a horizontal concentrated force $f_{x}^{\prime}$, a dislocation with vertical Burger's vector $b_{y}^{\prime}$, a center of shear along the $x$-direction equal to $\kappa_{x}$, and a double center of expansion along the $x$-direction equal to $\chi_{x}$. The solutions and magnitudes for the image singularities in (3.23) are

$$
\begin{aligned}
& \vec{\phi}^{f_{x}^{\prime}}=\frac{f_{x}^{\prime}}{\pi\left(1+k_{1}\right)}\left\{\sin \theta_{2} \mathbf{m}\left(\theta_{2}\right)+\left[\begin{array}{c}
\frac{1+k_{1}}{2} \theta_{2} \\
-\frac{1-k_{1}}{2} \ln r_{2}
\end{array}\right]\right\}, \\
& \vec{u}^{f_{x}^{\prime}}=\frac{f_{x}^{\prime}}{2 \pi \mu_{1}\left(1+k_{1}\right)}\left\{\sin \theta_{2} \mathbf{n}\left(\theta_{2}\right)-\left[\begin{array}{c}
k_{1} \ln r_{2} \\
0
\end{array}\right]\right\}, \quad f_{x}^{\prime}=\frac{2 \mu_{1} b_{y} v\left[\left(1+v k_{1}\right)-\left(v+k_{2}\right)\right]}{\left(v+k_{2}\right)\left(1+v k_{1}\right)} . \\
& \vec{\phi}^{b_{y}^{\prime}}=\frac{-2 \mu_{1} b_{y}^{\prime}}{\pi\left(1+k_{1}\right)}\left\{\sin \theta_{2} \mathbf{m}\left(\theta_{2}\right)-\left[\begin{array}{c}
0 \\
\ln r_{2}
\end{array}\right]\right\}, \\
& \vec{u}^{b_{y}^{\prime}}=\frac{-b_{y}^{\prime}}{\pi\left(1+k_{1}\right)}\left\{\sin \theta_{2} \mathbf{n}\left(\theta_{2}\right)+\left[\begin{array}{c}
\frac{1-k_{1}}{2} \ln r_{2} \\
-\frac{1+k_{1}}{2} \theta_{2}
\end{array}\right]\right\}, \quad b_{y}^{\prime}=\frac{\left(v^{2} k_{1}-k_{2}\right)}{\left(v+k_{2}\right)\left(1+v k_{1}\right)} b_{y} . \\
& \vec{\phi}^{k_{x}}=\frac{-2 \mu_{1} \kappa_{x}}{\pi\left(1+k_{1}\right) r_{2}} \cos 2 \theta_{2} \mathbf{m}\left(\theta_{2}\right), \quad \begin{array}{c}
\kappa_{x} \\
\vec{u}^{k_{x}}
\end{array}=\frac{\kappa_{x}}{2 \pi\left(1+k_{1}\right) r_{2}}\left\{\left[\begin{array}{c}
1-k_{1} \\
0 \quad k_{1}-1
\end{array}\right] \mathbf{n}\left(\theta_{2}\right)+2 \sin 2 \theta_{2} \mathbf{m}\left(\theta_{2}\right)\right\}, \\
& \vec{\phi}^{\chi_{x}}=\frac{4 \mu_{1} \chi_{x}}{\pi\left(1+k_{1}\right) r_{2}^{2}}\left(\sin \theta_{2} \mathbf{m}\left(\theta_{2}\right)-\cos \theta_{2} \mathbf{n}\left(\theta_{2}\right)\right), \quad k_{1} b_{y} h . \\
& \vec{u}^{\chi_{x}}=\frac{2 \chi_{x}}{\pi\left(1+k_{1}\right) r_{2}^{2}}\left(\cos \theta_{2} \mathbf{m}\left(\theta_{2}\right)+\sin \theta_{2} \mathbf{n}\left(\theta_{2}\right)\right), \quad \chi_{x}=\frac{(v-1)}{\left(1+v k_{1}\right)} b_{y} h^{2} .
\end{aligned}
$$

For material 2, the image singularities include a horizontal concentrated force $f_{x}^{\prime}$, a dislocation with vertical Burger's vector $b_{y}^{\prime}$, and a concentrate moment $M$. The stress function can be expressed as

$$
\overrightarrow{\phi^{i}}=\vec{\phi}^{f_{x}^{\prime}}+\vec{\phi}^{b_{y}^{\prime}}+\vec{\phi}^{M}
$$

and the solutions and magnitudes are

$$
\begin{aligned}
& \vec{\phi}^{f_{x}^{\prime}}=\frac{f_{x}^{\prime}}{\pi\left(1+k_{2}\right)}\left\{\sin \theta_{1} \mathbf{m}\left(\theta_{1}\right)+\left[\begin{array}{c}
\frac{1+k_{2}}{2} \theta_{1} \\
-\frac{1-k_{2}}{2} \ln r_{1}
\end{array}\right]\right\}, \\
& \vec{u}^{f_{x}^{\prime}}=\frac{f_{x}^{\prime}}{2 \pi \mu_{2}\left(1+k_{2}\right)}\left\{\sin \theta_{1} \mathbf{n}\left(\theta_{1}\right)-\left[\begin{array}{c}
k_{2} \ln r_{1} \\
0
\end{array}\right]\right\}, \quad f_{x}^{\prime}=\frac{2 \mu_{2} b_{y}\left[\left(v+k_{2}\right)-\left(1+v k_{1}\right)\right]}{\left(v+k_{2}\right)\left(1+v k_{1}\right)} . \\
& \vec{\phi}^{b_{y}^{\prime}}=\frac{-2 \mu_{2} b_{y}^{\prime}}{\pi\left(1+k_{2}\right)}\left\{\sin \theta_{1} \mathbf{m}\left(\theta_{1}\right)-\left[\begin{array}{c}
0 \\
\ln r_{1}
\end{array}\right]\right\}, \\
& \vec{u}^{b_{y}^{\prime}}=\frac{-b_{y}^{\prime}}{\pi\left(1+k_{2}\right)}\left\{\sin \theta_{1} \mathbf{n}\left(\theta_{1}\right)+\left[\begin{array}{c}
\frac{1-k_{2}}{2} \ln r_{1} \\
-\frac{1+k_{2}}{2} \theta_{1}
\end{array}\right]\right\}, \quad b_{y}^{\prime}=\frac{\left[\left(v+k_{2}\right)+k_{2}\left(1+v k_{1}\right)\right]}{\left(v+k_{2}\right)\left(1+v k_{1}\right)} b_{y} .
\end{aligned}
$$




$$
\vec{\phi}^{M}=\frac{M}{\pi r_{1}} \mathbf{m}\left(\theta_{1}\right), \quad \vec{u}^{M}=\frac{M}{2 \pi \mu_{2} r_{1}} \mathbf{n}\left(\theta_{1}\right), \quad M=\frac{2 \mu_{2} b_{y} h\left[\left(v+k_{2}\right)-\left(1+v k_{1}\right)\right]}{\left(v+k_{2}\right)\left(1+v k_{1}\right)} .
$$

\subsection{Remarks}

(1) The structure of image singularities for applied concentrated forces and dislocations are similar. For material 1 , there are four image singularities at the image point $(0,-h)$. Two of them have a stress singularities with $\mathrm{O}(1 / r)$, one of them has a stress singularity with $\mathrm{O}\left(1 / r^{2}\right)$ and the remaining one has a stress singularity with $\mathrm{O}\left(1 / r^{3}\right)$. For material 2 , there are three image singularities at the image point $(0, h)$. Two of them have stress singularities with $\mathrm{O}(1 / r)$ and the other has a stress singularity with $\mathrm{O}\left(1 / r^{2}\right)$. It is to be noted that there is no image singularity with $\mathrm{O}\left(1 / r^{3}\right)$ for material 2 . The magnitude of higher order image singularities, i.e. $\mathrm{O}\left(1 / r^{2}\right)$ and $\mathrm{O}\left(1 / r^{3}\right)$, are proportional to $h$ and $h^{2}$ respectively. As the applied singularity at the object point approaches the interface, i.e. $h \rightarrow 0$, all the higher order image singularities disappear and only concentrated forces and dislocations with stress singularity of $\mathrm{O}(1 / r)$ remain.

(2) The resultant forces and dislocations for image singularities of the bimaterials are represented in the following matrix forms. For material 1, the result is

$$
\left[\begin{array}{l}
f_{x}^{r} \\
f_{y}^{r} \\
b_{x}^{r} \\
b_{y}^{r}
\end{array}\right]_{1}=\left[\begin{array}{cccc}
a & 0 & 0 & -c \\
0 & a & c & 0 \\
0 & b & -a & 0 \\
-b & 0 & 0 & -a
\end{array}\right]\left[\begin{array}{l}
f_{x} \\
f_{y} \\
b_{x} \\
b_{y}
\end{array}\right]
$$

and for material 2, the result is

$$
\left[\begin{array}{l}
f_{x}^{r} \\
f_{y}^{r} \\
b_{x}^{r} \\
b_{y}^{r}
\end{array}\right]_{2}=\left[\begin{array}{cccc}
d & 0 & 0 & c \\
0 & d & -c & 0 \\
0 & -b & e & 0 \\
b & 0 & 0 & e
\end{array}\right]\left[\begin{array}{l}
f_{x} \\
f_{y} \\
b_{x} \\
b_{y}
\end{array}\right],
$$

where

$$
\begin{aligned}
& a=\frac{\left(k_{2}-v^{2} k_{1}\right)}{\left(v+k_{2}\right)\left(1+v k_{1}\right)}, \quad b=\frac{\left[k_{1}\left(v+k_{2}\right)-k_{2}\left(1+v k_{1}\right)\right]}{2 \mu_{1}\left(v+k_{2}\right)\left(1+v k_{1}\right)}, \quad c=\frac{2 \mu_{2}\left[\left(v+k_{2}\right)-\left(1+v k_{1}\right)\right]}{\left(v+k_{2}\right)\left(1+v k_{1}\right)}, \\
& d=\frac{v\left[\left(1+v k_{1}\right)+k_{1}\left(v+k_{2}\right)\right]}{\left(v+k_{2}\right)\left(1+v k_{1}\right)}, \quad e=\frac{\left[k_{2}\left(1+v k_{1}\right)+\left(v+k_{2}\right)\right]}{\left(1+v k_{1}\right)\left(v+k_{2}\right)} .
\end{aligned}
$$

From (3.34), it is noted that $a+d=1$ and $e-a=1$. From (3.32) and (3.33) and for the case of applied concentrated force $f_{x}$ acting in material 1 , the force and dislocation for the image singularities are $\left(f_{x}^{r}\right)_{1}=a f_{x}$ and $\left(b_{y}^{r}\right)_{1}=-b f_{x}$ for material 1 , and $\left(f_{x}^{r}\right)_{2}=d f_{x}$ and $\left(b_{y}^{r}\right)_{2}=b f_{x}$ for material 2. However, the resultant force for the image singularities of materials 1 and 2 taken together is $\left(f_{x}^{r}\right)_{1}+\left(f_{x}^{r}\right)_{2}=$ $(a+d) f_{x}=f_{x}$, which is identical to the applied force, while the net dislocation for the image singularities is zero, i.e. $\left(b_{y}^{r}\right)_{1}+\left(b_{y}^{r}\right)_{2}=0$. Similarly, the resultant dislocation for the image singularities of materials 1 and 2 due to a dislocation $b_{x}$ acting in material 1 is $\left(b_{x}^{r}\right)_{1}+\left(b_{x}^{r}\right)_{2}=(e-d) b_{x}=b_{x}$, which is identical to the applied dislocation, and the net force is zero. Hence, it is interesting to conclude that the resultant forces or dislocations for the image singularities of the bimaterial are identical to the applied forces and dislocations, respectively.

(3) For the special case of a half-plane with traction-free boundary condition, we have

$$
a=1, \quad b=\frac{k_{1}-1}{2 \mu_{1}}, \quad c=0, \quad d=0, \quad \text { and } \quad e=2 .
$$


And for the case of a half-plane with rigidly fixed boundary, these quantities becomes

$$
a=-1, \quad b=0, \quad c=\frac{2 \mu_{1}\left(1-k_{1}\right)}{k_{1}}, \quad d=2, \quad \text { and } \quad e=0 .
$$

The resultant forces and dislocations for the special cases of the half-plane are obtained from (3.34) by setting $v=0$ for the traction-free boundary, and $v=\infty$ for the rigidly fixed boundary. The corresponding results for the anisotropic case of the half-plane have been obtained by Ting (1996).

\section{Representation of image singularities in matrix form}

The complete structure of image singularities for applied forces and dislocations has been determined and discussed in the previous sections. In order to simplify these results, a representation of the solutions in matrix forms is instructive. The behavior of all the image singularities obtained in this study involves three different orders of stress singularity, which are (a) concentrated force and dislocation with stress singularities of $\mathrm{O}(1 / r)$; (b) double force, center of shear, center of extension, center of expansion, concentrated moment and center of dilatation with stress singularities of $\mathrm{O}\left(1 / r^{2}\right)$; double center of expansion and double moment with stress singularities of $\mathrm{O}\left(1 / r^{3}\right)$. However all the solutions for higher order image singularities (i.e $\mathrm{O}\left(1 / r^{2}\right)$ and $\mathrm{O}\left(1 / r^{3}\right)$ ) can be obtained by differentiation of the solutions for concentrated forces and dislocations. We define the stress function $\vec{\varphi}$ such that

$$
\vec{\varphi}^{T}=\vec{\phi}^{T} / M
$$

where the superscript $T$ indicates the type of singularity and $M$ is the magnitude of the singularity.

For instance, if $T \equiv f_{y}$ or $T \equiv b_{x}$, we have

$$
\vec{\varphi}^{f_{y}}=\left(\varphi_{x}^{f_{y}}, \varphi_{y}^{f_{y}}\right)=\left(\phi_{x}^{f_{y}}, \phi_{y}^{f_{y}}\right) / f_{y},
$$

or

$$
\vec{\varphi}^{b_{x}}=\left(\varphi_{x}^{b_{x}}, \varphi_{y}^{b_{x}}\right)=\left(\phi_{x}^{b_{x}}, \phi_{y}^{b_{x}}\right) / b_{x} .
$$

The stress functions for the higher order image singularities can be represented by the solutions of concentrated forces and dislocations as

$$
\begin{aligned}
& \vec{\varphi}^{P_{x}}=-\frac{\partial \vec{\varphi}^{f_{x}}}{\partial x}, \quad \vec{\varphi}^{C_{x}}=-\frac{\partial \vec{\varphi}^{f_{y}}}{\partial x}, \\
& \vec{\varphi}^{M}=-\left(\frac{\partial \vec{\varphi}^{f_{y}}}{\partial x}+\frac{\partial \vec{\varphi}^{f_{x}}}{\partial y}\right), \quad \vec{\varphi}^{D}=-\left(\frac{\partial \vec{\varphi}^{f_{x}}}{\partial x}+\frac{\partial \vec{\varphi}^{f_{y}}}{\partial y}\right), \\
& \vec{\varphi}^{\gamma_{x}}=-\frac{\partial \vec{\varphi}^{b_{y}}}{\partial x}, \quad \vec{\varphi}^{\kappa_{x}}=-\frac{\partial \vec{\varphi}^{b_{x}}}{\partial x}, \vec{\varphi}^{\chi}=-\left(\frac{\partial \vec{\varphi}^{b_{y}}}{\partial x}+\frac{\partial \vec{\varphi}^{b_{x}}}{\partial y}\right), \\
& \vec{\varphi}^{M_{x}}=\frac{\partial}{\partial x}\left(\frac{\partial \vec{\varphi}^{f_{y}}}{\partial x}+\frac{\partial \vec{\varphi}^{f_{x}}}{\partial y}\right), \quad \vec{\varphi}^{M_{y}}=\frac{\partial}{\partial y}\left(\frac{\partial \vec{\varphi}^{f_{y}}}{\partial x}+\frac{\partial \vec{\varphi}^{f_{x}}}{\partial y}\right), \\
& \vec{\varphi}^{\chi_{x}}=\frac{\partial}{\partial x}\left(\frac{\partial \vec{\varphi}^{b_{y}}}{\partial x}+\frac{\partial \vec{\varphi}^{b_{x}}}{\partial y}\right), \quad \vec{\varphi}^{\chi_{y}}=\frac{\partial}{\partial y}\left(\frac{\partial \vec{\varphi}^{b_{y}}}{\partial x}+\frac{\partial \vec{\varphi}^{b_{x}}}{\partial y}\right) .
\end{aligned}
$$


Hence, the fundamental solutions we need for constructing all the image singularities for the bimaterials are only the solutions for concentrated forces and dislocations in an infinite plane. Finally, all the results of image singularities that we have obtained in the previous sections can be summarized and represented in the following compact matrix form. The image singularities for material 1 are

$$
\left[\vec{\phi}^{i}\right]=[Q]\left\{\left[O_{1}\right]\left[\begin{array}{c}
\varphi^{f_{x}} \\
\varphi^{f_{y}} \\
\varphi^{b_{x}} \\
\varphi^{b_{y}}
\end{array}\right]+h\left[O_{2}\right]\left[\begin{array}{c}
-\frac{\partial \varphi^{f_{x}}}{\partial x} \\
-\frac{\partial \varphi^{f_{y}}}{\partial x} \\
-\frac{\partial \varphi^{b_{x}}}{\partial x} \\
-\frac{\partial \varphi^{b_{y}}}{\partial x}
\end{array}\right]+h^{2}\left[O_{3}\right]\left[\begin{array}{c}
\frac{\partial}{\partial x}\left(\frac{\partial \varphi^{f_{y}}}{\partial x}+\frac{\partial \varphi^{f_{x}}}{\partial y}\right) \\
\frac{\partial}{\partial x}\left(\frac{\partial \varphi^{f_{y}}}{\partial x}+\frac{\partial \varphi^{f_{x}}}{\partial y}\right) \\
\frac{\partial}{\partial x}\left(\frac{\partial \varphi^{b_{y}}}{\partial x}+\frac{\partial \varphi^{b_{x}}}{\partial y}\right) \\
\frac{\partial}{\partial x}\left(\frac{\partial \varphi^{b_{y}}}{\partial x}+\frac{\partial \varphi^{b_{x}}}{\partial y}\right)
\end{array}\right]\right\}
$$

where $[Q]=\left[\begin{array}{llll}f_{x} & f_{y} & 2 \mu_{1} b_{x} & 2 \mu_{1} b_{y}\end{array}\right]$ and

$$
\begin{aligned}
& {\left[O_{1}\right]=\left[\begin{array}{cccc}
\frac{k_{2}-v^{2} k_{1}}{\left(1+v k_{1}\right)\left(v+k_{2}\right)} & 0 & 0 & \frac{k_{2}\left(1+v k_{1}\right)-k_{1}\left(v+k_{2}\right)}{\left(1+v k_{1}\right)\left(v+k_{2}\right)} \\
0 & \frac{k_{2}-v^{2} k_{1}}{\left(1+v k_{1}\right)\left(v+k_{2}\right)} & -\frac{k_{2}\left(1+v k_{1}\right)-k_{1}\left(v+k_{2}\right)}{\left(1+v k_{1}\right)\left(v+k_{2}\right)} & 0 \\
0 & \frac{v\left(\left(1+v k_{1}\right)-\left(v+k_{2}\right)\right)}{\left(1+v k_{1}\right)\left(v+k_{2}\right)} & \frac{v^{2} k_{1}-k_{2}}{\left(1+v k_{1}\right)\left(v+k_{2}\right)} & 0 \\
\frac{v\left(\left(1+v k_{1}\right)-\left(v+k_{2}\right)\right)}{\left(1+v k_{1}\right)\left(v+k_{2}\right)} & 0 & 0 & \frac{v^{2} k_{1}-k_{2}}{\left(1+v k_{1}\right)\left(v+k_{2}\right)}
\end{array}\right],} \\
& {\left[O_{2}\right]=\left[\begin{array}{cccc}
0 & \frac{2(1-v)}{1+v k_{1}} & 0 & 0 \\
-\frac{2(1-v)}{1+v k_{1}} & 0 & 0 & 0 \\
0 & 0 & 0 & \frac{2(1-v)}{1+v k_{1}} \\
0 & 0 & \frac{2(1-v)}{1+v k_{1}} & 0
\end{array}\right]} \\
& {\left[O_{3}\right]=\left[\begin{array}{cccc}
0 & \frac{2(1-v)}{\left(1+k_{1}\right)\left(1+v k_{1}\right)} & 0 & 0 \\
\frac{2(1-v)}{\left(1+k_{1}\right)\left(1+v k_{1}\right)} & 0 & 0 & 0 \\
0 & 0 & 0 & \frac{(v-1)}{\left(1+v k_{1}\right)} \\
0 & 0 & \frac{2(1-v)}{\left(1+k_{1}\right)\left(1+v k_{1}\right)} 0
\end{array}\right] .}
\end{aligned}
$$


The image singularities for material 2 are

$$
\left[\overrightarrow{\phi^{i}}\right]=\left[Q^{*}\right]\left\{\left[O_{1}^{*}\right]\left[\begin{array}{c}
\varphi^{f_{x}} \\
\varphi^{f_{y}} \\
\varphi^{b_{x}} \\
\varphi^{b_{y}}
\end{array}\right]+h\left[O_{2}^{*}\right]\left[\begin{array}{c}
-\left(\frac{\partial \varphi^{f_{y}}}{\partial x}+\frac{\partial \varphi^{f_{x}}}{\partial y}\right) \\
-\left(\frac{\partial \varphi^{f_{x}}}{\partial x}+\frac{\partial \varphi^{f_{y}}}{\partial y}\right) \\
-\left(\frac{\partial \varphi^{b_{y}}}{\partial x}+\frac{\partial \varphi^{b_{x}}}{\partial y}\right) \\
-\left(\frac{\partial \varphi^{b_{y}}}{\partial x}+\frac{\partial \varphi^{b_{x}}}{\partial y}\right)
\end{array}\right]\right\},
$$

where $\left[Q^{*}\right]=\left[\begin{array}{lllll}f_{x} & f_{y} & 2 \mu_{2} b_{x} & 2 \mu_{2} b_{y}\end{array}\right]$ and

$$
\begin{gathered}
{\left[O_{1}^{*}\right]=\left[\begin{array}{cccc}
\frac{v\left(\left(1+v k_{1}\right)+k_{1}\left(v+k_{2}\right)\right)}{\left(1+v k_{1}\right)\left(v+k_{2}\right)} & 0 & 0 & \frac{v\left(k_{2}\left(1+v k_{1}\right)-k_{1}\left(v+k_{2}\right)\right)}{\left(1+v k_{1}\right)\left(v+k_{2}\right)} \\
0 & \frac{v\left(\left(1+v k_{1}\right)+k_{1}\left(v+k_{2}\right)\right)}{\left(1+v k_{1}\right)\left(v+k_{2}\right)} & -\frac{v\left(k_{2}\left(1+v k_{1}\right)-k_{1}\left(v+k_{2}\right)\right)}{\left(1+v k_{1}\right)\left(v+k_{2}\right)} & 0 \\
0 & \frac{v\left(\left(1+v k_{1}\right)-\left(v+k_{2}\right)\right)}{\left(1+v k_{1}\right)\left(v+k_{2}\right)} & \frac{\left(1+v k_{1}\right)+k_{1}\left(v+k_{2}\right)}{\left(1+v k_{1}\right)\left(v+k_{2}\right)} & 0 \\
-\frac{v\left(\left(1+v k_{1}\right)-\left(v+k_{2}\right)\right)}{\left(1+v k_{1}\right)\left(v+k_{2}\right)} & 0 & 0 & -\frac{\left(1+v k_{1}\right)+k_{1}\left(v+k_{2}\right)}{\left(1+v k_{1}\right)\left(v+k_{2}\right)}
\end{array}\right],} \\
{\left[O_{2}^{*}\right]=\left[\begin{array}{cccc}
\frac{v\left(\left(1+v k_{1}\right)-\left(v+k_{2}\right)\right)}{\left(1+v k_{1}\right)\left(v+k_{2}\right)} \\
0 & -\frac{v\left(1+k_{2}\right)\left(\left(1+v k_{1}\right)-\left(v+k_{2}\right)\right)}{\left(1+k_{2}\right)\left(1+v k_{1}\right)\left(v+k_{2}\right)} & 0 & 0 \\
0 & 0 & \frac{v\left(1+k_{2}\right)\left(\left(1+v k_{1}\right)-\left(v+k_{2}\right)\right)}{\left(1+v k_{1}\right)\left(v+k_{2}\right)} & 0 \\
0 & 0 & 0 & -\frac{v\left(\left(1+v k_{1}\right)-\left(v+k_{2}\right)\right)}{\left(1+v k_{1}\right)\left(v+k_{2}\right)}
\end{array}\right] .}
\end{gathered}
$$

\section{Concluding remarks}

The structures of image singularities for bimaterials have been obtained and discussed in detail in this study. It shows that the Green's function for the bimaterials consists of several Green's functions for the homogeneous infinite plane subjected to various types of singularities applied at the object and image points. This is useful information that puts the solution in a different perspective. The image singularities for different types of applied singularity in material 1 for bimaterials are summarized and listed in Tables 2 and 3. It is interesting to note that the image singularities for concentrated forces are not only force systems but also dislocations. In addition, the image singularities of dislocations also include force systems. The image singularities consist of concentrated forces and dislocations with $\mathrm{O}(1 / r)$, together with higher order singularities $\left(\mathrm{O}\left(1 / r^{2}\right)\right.$ and $\left.\mathrm{O}\left(1 / r^{3}\right)\right)$ for isotropic bimaterials. However, the image singularities for anisotropic bimaterials are simply concentrated forces and dislocations, the location of the image point being different for each image singularity, depending on the material constants (Ting, 1996).

It is shown in this paper that the solutions for higher order singularities can be derived by differentiating the basic solutions for the concentrated forces and dislocations. Hence, the fundamental solutions required for constructing the image singularities only those corresponding to the concentrated force and dislocation in an infinite plane. All the image singularities of bimaterials are represented in a compact matrix form in terms of the solutions of concentrated forces and dislocations. It is noted that the image singularities of material 1 have stress singularities with $\mathrm{O}(1 / r), \mathrm{O}\left(1 / r^{2}\right)$ and $\mathrm{O}\left(1 / r^{3}\right)$; but stress singularities with $\mathrm{O}(1 / r)$ 
Table 2

Applied singularities (concentrated forces and dislocations) and the correspondent image singularities for material 1

\begin{tabular}{|c|c|c|c|c|}
\hline \multirow[t]{2}{*}{ Image singularity } & \multicolumn{4}{|c|}{ Applied singularity } \\
\hline & $f_{x}$ & $f_{y}$ & $b_{x}$ & $b_{y}$ \\
\hline Horizontal body-force $f_{x}^{\prime}$ & $\Delta$ & & & $\Delta$ \\
\hline Vertical body-force $f_{y}^{\prime}$ & & $\Delta$ & $\Delta$ & \\
\hline Dislocation $b_{x}^{\prime}$ & & $\Delta$ & $\Delta$ & \\
\hline Dislocation $b_{y}^{\prime}$ & $\Delta$ & & & $\Delta$ \\
\hline Double force without moment along the $x$-direction, $P_{x}$ & & $\Delta$ & & \\
\hline Double force with moment along the $x$-direction, $C_{x}$ & $\Delta$ & & & \\
\hline Center of extension along the $x$-direction, $\gamma_{x}$ & & & $\Delta$ & \\
\hline Center of shear along the $x$-direction, $\kappa_{x}$ & & & & $\boldsymbol{\Delta}$ \\
\hline Double center-of-expansion along the $x$-direction, $\chi_{x}$ & & & & $\boldsymbol{\Delta}$ \\
\hline Double center-of-expansion along the $y$-direction, $\chi_{y}$ & & & $\Delta$ & \\
\hline Double moment along the $x$-direction, $M_{x}$ & & $\Delta$ & & \\
\hline Double moment along the $y$-direction, $M_{y}$ & $\boldsymbol{\Delta}$ & & & \\
\hline
\end{tabular}

Table 3

Applied singularities (concentrated forces and dislocations) and the correspondent image singularities for material 2

\begin{tabular}{|c|c|c|c|c|}
\hline \multirow[t]{2}{*}{ Image singularity } & \multicolumn{4}{|c|}{ Applied singularity } \\
\hline & $f_{x}$ & $f_{y}$ & $b_{x}$ & $b_{y}$ \\
\hline Horizontal body force, $f_{x}^{\prime}$ & $\Delta$ & & & $\Delta$ \\
\hline Vertical body force, $f_{y}^{\prime}$ & & $\Delta$ & $\Delta$ & \\
\hline Dislocation, $b_{x}^{\prime}$ & & $\Delta$ & $\Delta$ & \\
\hline Dislocation, $b_{y}^{\prime}$ & $\Delta$ & & & $\Delta$ \\
\hline Center of dilatation, $D$ & & $\Delta$ & & \\
\hline Concentrated moment, $M$ & $\Delta$ & & & $\Delta$ \\
\hline Center of expansion, $\chi$ & & & $\Delta$ & \\
\hline
\end{tabular}

and $\mathrm{O}\left(1 / r^{2}\right)$ only exist for the image singularities of material 2 . The magnitudes of higher order image singularities, i.e. $\mathrm{O}\left(1 / r^{2}\right)$ and $\mathrm{O}\left(1 / r^{3}\right)$, are proportional to $h$ and $h^{2}$ respectively. As the applied concentrated forces or dislocations approach the interface, the higher order image singularities disappear, and only the image singularities with $\mathrm{O}(1 / r)$, which correspond to concentrated forces and dislocations, remain in the final solution. Based on the results obtained in this study, the image singularities for higher order applied singularities (namely double force, center of shear, etc.) for bimaterials can be easily derived.

\section{Acknowledgements}

The financial support of the authors from the National Science Council, Republic of China, through Grant NSC 87-2218-E002-022 to National Taiwan University is gratefully acknowledged.

\section{References}

Barnett, D.M., Lothe, J., 1974. An image force theorem for dislocations in bicrystals. Journal of Physics 4, $1618-1635$.

Carvalho, J.L., Curran, J.H., 1992. Two-dimensional Green's functions for elastic bimaterials. ASME Journal of Applied Mechanics 59, 321-327. 
Dundurs, J., Hetenyi, M., 1965. Transmission of force between two semi-infinite solids. ASME Journal of Applied Mechanics 32, 671674.

Hasegawa, H., Lee, V.G., Mura, T., 1992. Green's functions for axisymmetric problems of dissimilar elastic solids. ASME Journal of Applied Mechanics 59, 312-319.

Hwu, Chyanbin, Yen, Wen J., 1991. Green's functions of two-dimensional anisotropic plates containing an elliptic hole. International Journal of Solids Structures 27, 1705-1719.

Ma, C.C., Huang, K.C., 1996. Exact transient solutions of buried dynamic point forces for elastic bimaterials. International Journal of Solids and Structures 33, 4511-4529.

Ma, C.C., Lin, R.L., 2001. Image singularities of Green's functions for an isotropic elastic half-plane subjected to forces and dislocations. Mathematics and Mechanics of Solids 6, 503-524.

Mindlin, R.D., 1936. Force at a point in the interior of a semi-infinite solid. Physics 7, 195-202.

Mindlin, R.D., Cheng, D.H., 1950. Nuclei of strain in the semi-infinite solid. Journal of Applied Physics 21, 926-930.

Phan-Thien, N., 1983. On the image system for the Kelvin-state. Journal of Elasticity 13, 231-235.

Jianmin, Qu, Qiangian, Li, 1991. Interfacial dislocation and its application to interface crack in anisotropic bimaterials. Journal of Elasticity 26, 167-195.

Suo, Z., 1990. Singularities, interfaces and cracks in dissimilar anisotropic media. Proceedings of The Royal Society of London A 427, 331-358.

Ting, T.C., 1992. Image singularities of Green's functions for anisotropic elastic half-space and bimaterials. The Quarterly Journal of Mechanics and Applied Mathematics 45, 119-139.

Ting, T.C., 1996. In: Anisotropic Elasticity. New York Press, Oxford, pp. 247-311.

Vijayakumar, S., Cormack, D.E., 1987a. Green's functions for the biharmonic equation: bonded elastic media. SIAM Journal of Applied Mathematics 47, 982-997.

Vijayakumar, S., Cormack, D.E., 1987b. Nuclei of strain for bi-material elastic media with sliding interface. Journal of Elasticity 17, 285-290.

Willis, J.R., 1970. Stress field produced by dislocations in anisotropic media. Philosophical Magazine 21, 931-949. 